\title{
Lepidoptera of Arkhangelsk oblast of Russia: a regional checklist
}

\author{
Mikhail V. Kozlov, Jaakko Kullberg \& Vitali E. Zverev
}

Kozlov, M. V., Kullberg, J. \& Zverev, V. E. 2014: Lepidoptera of Arkhangelsk oblast of Russia: a regional checklist. — Entomol. Fennica 25: 113-141.

The first regional checklist of moths and butterflies of Arkhangelsk oblast of Russia includes 1,036 species ( 538 species of microlepidoptera and 498 species of macrolepidoptera), 496 of which have been found in the oblast for the first time. The most interesting records include Gnorimoschema robustella, Caryocolum leucomelanella, Dichrorampha sequana, D. uralensis, Neptis rivularis and Melitaea phoebe. We also discovered several populations of Parnassius mnemosyne in the southern part of the oblast. The fauna of Arkhangelsk oblast appears poorer than the fauna of Northern Ural Mts. but still includes some Siberian taiga species which do not reach Fennoscandia. Also, the distribution limits of several species extend further north in Arkhangelsk oblast than in the more western parts of Europe. We estimate that 500 to 800 species remain to be found in the study region.

M. V. Kozlov, Section of Ecology, University of Turku, FI-20014 Turku, Finland; E-mail:mikoz@utu.fi

J. Kullberg, Finnish Museum of Natural History, Zoology Unit, University of Helsinki, P.O. Box 17, FI-00014 Helsinki, Finland; E-mail: jkullber@mappi. helsinki.fi

V. E. Zverev, Section of Ecology, University of Turku, FI-20014 Turku, Finland; E-mail:vitzve@utu.fi

Received 29 January 2014, accepted 29 April 2014

\section{Introduction}

Arkhangelsk oblast covers an area of 587,400 $\mathrm{km}^{2}$, slightly larger than France, and is located in the northern part of the East European Plain. It includes Nenets Autonomous Okrug (NAO), the continental part of which is flat lowland tundra (Bolshezemelskaya Tundra) with several hill chains, while the Arctic islands (Novaya Zemlya and Franz Joseph Land) are mountainous. Due to the obvious environmental and biogeographical distinction between NAO and the remaining (mostly forested) parts of Arkhangelsk oblast, and because of an insufficient knowledge on the moths and butterflies of NAO, we deliberately excluded NAO from our study region. Thus, the area covered by our checklist (Fig. 1) is 410,700 $\mathrm{km}^{2}$. For the reasons of brevity, hereafter "Arkhangelsk oblast" means "Arkhangelsk oblast excluding NAO”.

The northernmost parts of Arkhangelsk oblast (Mezensky district and the northern part of Primorskyj district; Fig. 1) is forest-tundra, whereas the remaining territory is covered by boreal taiga forests. Arbitrarily, these forests are subdivided into northern taiga (to the north of 64 


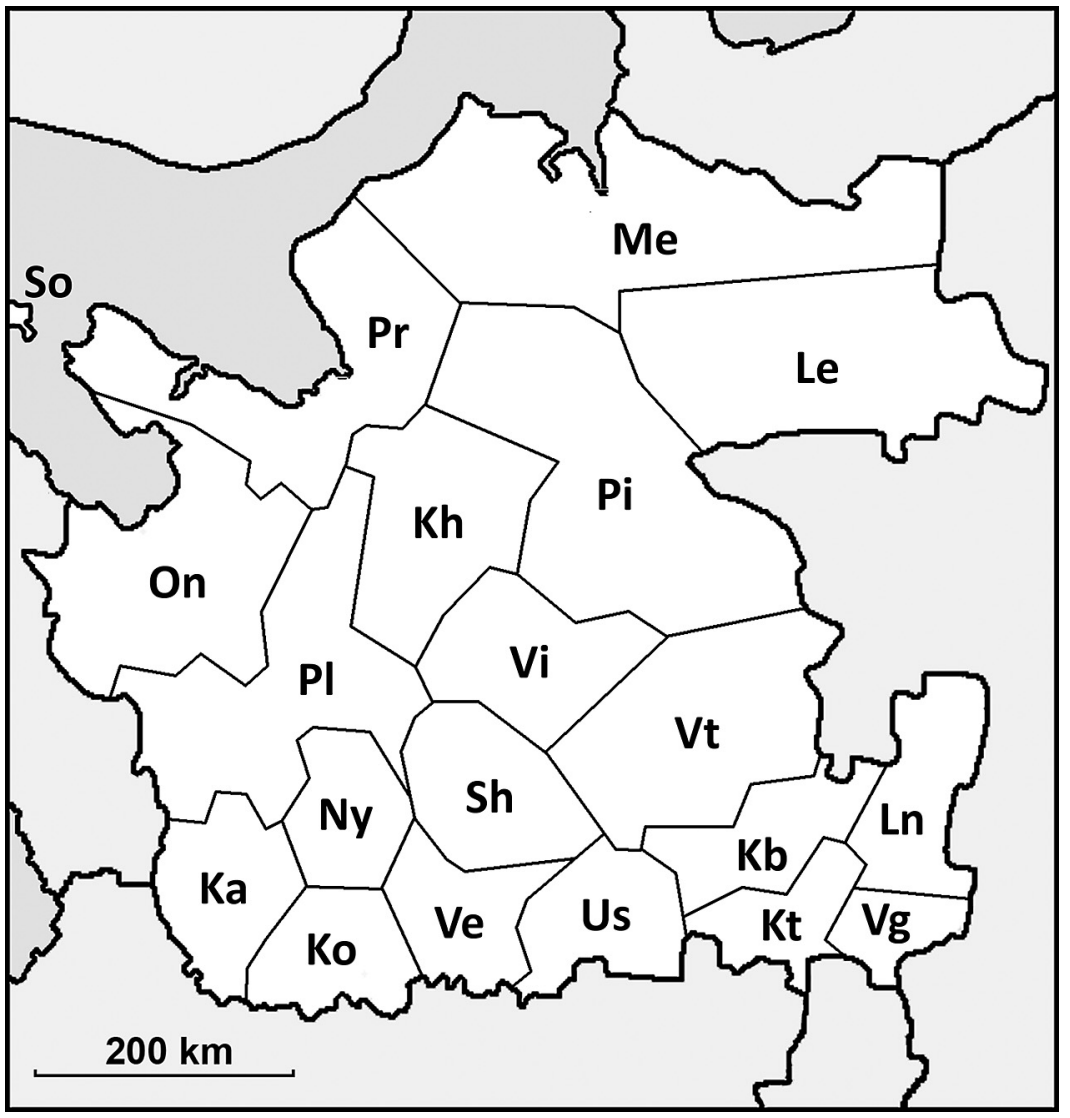

Fig. 1. Districts of Arkhangelsk oblast (excluding Nenets Autonomous Okrug): $\mathrm{Ka}-$ Kargopolsky, Kh Kholmogorsky, Ko Konoshsky, Kt - Kotlassky, Kb - Krasnoborsky, Ln - Lensky, Le - Leshukonsky, Me Mezensky, Ny - Nyandomsky, On - Onezhsky, Pi - Pinezhsky, PI - Plesetsky, Pr - Primorsky, Sh - Shenkursky, So - Solovetsky Archipelago (belongs to Pr), Us - Ustjansky, Ve - Velsky, Vt - Verkhnetotemsky, $\mathrm{Vg}-$ Vilegotsky, Vi - Vinogradovsky.

$65^{\text {th }}$ latitudes), middle taiga (most of the oblast), and southern taiga (Konoshsky district). Nearly two thirds of forests are formed by Norway spruce, the remaining forests are dominated by Scots pine (20\%) and birches (14\%). Typical Siberian trees (Abies sibirica, Larix sibirica), which do not belong to the recent flora of the Nordic countries, occupy relatively small areas to the East of Onega River. Wetlands cover 14\% of Arkhangelsk oblast, and meadows $2.5 \%$. The most fertile meadows are located on flood-plains along Northern Dvina and Mezen Rivers (Byzova 2007).

One of the most fascinating regions of Arkhangelsk oblast is the Kuloi plateau on the White Sea confined by the rivers of the Northern Dvina, the Pinega and the Kuloi. In this region, gypsum and anhydrite rocks of the Early Permian age are subjected to active karst processes. One of the flagship plants of this region is Paeonia anomala. The strongly meandering rivers have remarkable impacts on diversity of the riparian biotopes and act as migration paths for the southern fauna and flora (Mazur \& Kubisz 2013).

The insect fauna of Arkhangelsk oblast is poorly known. The two first reports on Lepidoptera from this region listed 126 species collected in Kotlas (Krulikovsky 1906) and 13 species collected mostly in Mezensky district (Poppius 1906). The next paper by Krulikovsky (1909) increased the number of species recorded in Kotlas to 280 . From late 1960 s to mid-1970s, L. F. Zelenova intensively investigated moths feeding on trees and shrubs in the city of Arkhangelsk and its surrounding areas. Her major publication (Zelenova 1972) reports 201 species, and several more species were added in subsequent publications (Zelenova 1973, 1976). Importantly, L. F. Zelenova worked under supervision of V. I. Kuznetzov, who checked many of her identifications. From 1991 to 1994, A. M. Tikhomirov collected macrolepidoptera in the Pinega reserve. Results of his work are partially published (Tikhomirov 1994, Tikhomirov \& Bolotov 2000, 
Fig. 2. Sampling localities (dots) in the Arkhangelsk oblast. Urban areas are shown by squares.

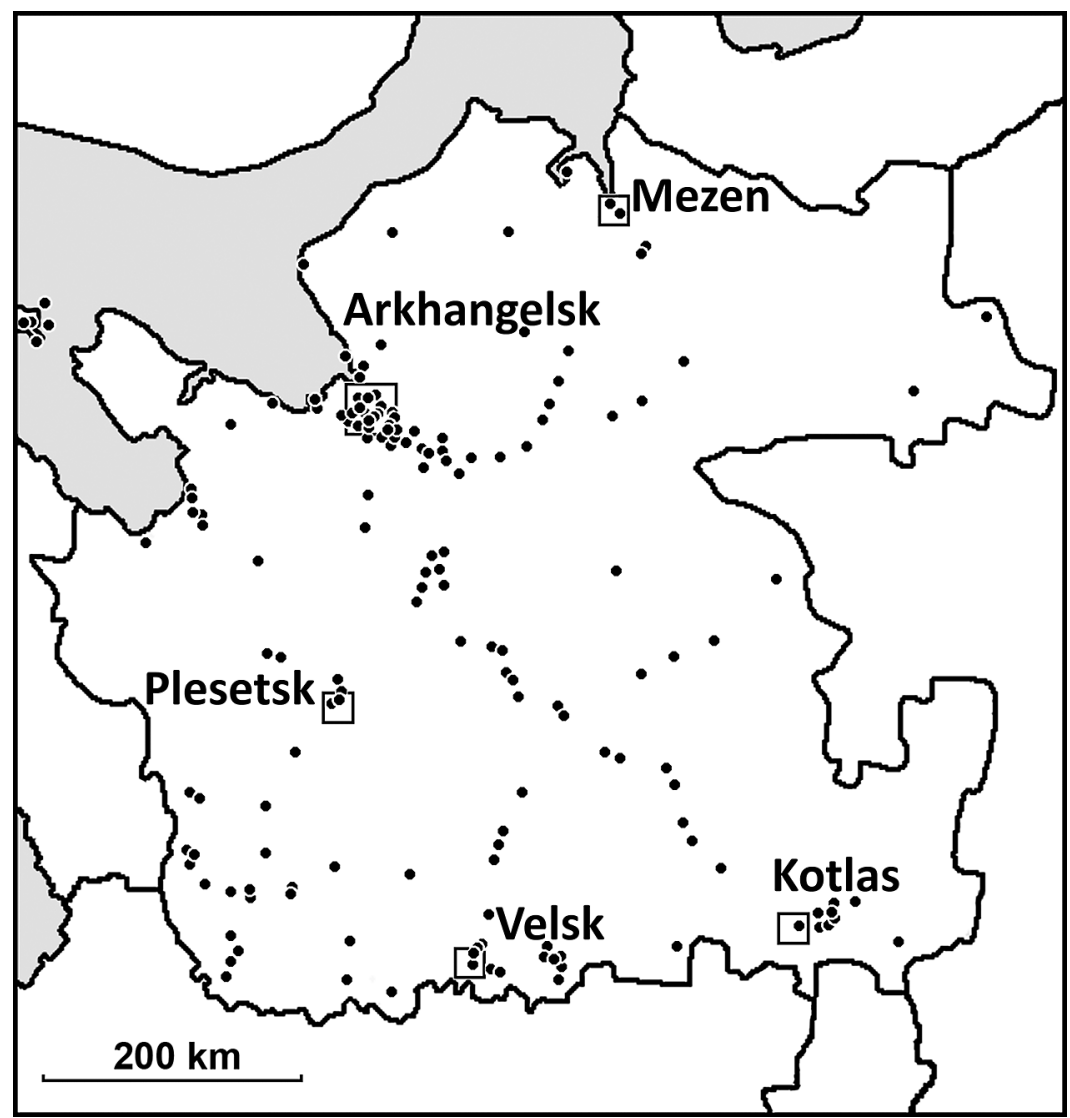

Antonova \& Tikhomirov 2002). From the mid1990s, I. N. Bolotov has been actively studying butterflies of Arkhangelsk oblast (Bolotov 2002, Bolotov et al. 2013a). Some information on macrolepidoptera was published in the lists of rare and protected species (Bolotov et al. 1998, Bolotov \& Semushin 2003) as well as in two papers on Parnassius mnemosyne (Linnaeus) (Rykov 2009, Bolotov et al. 2013b). Finally, the rarely observed, but widespread palaearctic tiger moth, Borearctia menetriesii (Eversmann), was recently discovered in the study region (Bolotov et al. 2013c).

Although several applied publications (Ezhov 2008, Ezhov \& Burak 2010, Burak \& Ezhov 2011) reported records of some moth species in Arkhangelsk oblast, identifications of these species are doubtful and therefore these data sources were not used while compiling the checklist.

In this paper we summarize the recent knowledge on moths and butterflies of Arkhangelsk oblast in a form of checklist reporting localities from which each species was collected. We believe that, in spite of the obvious incompleteness, this list may appear useful for ecological and biographical research and will facilitate further studies of Lepidoptera in the north-eastern Europe through identification of taxonomic and geographic gaps in our knowledge.

\section{Material and methods}

This paper is primarily based on the materials (some 4,500 pinned specimens, selected from over 17,500 collected specimens) sampled by M.V.K. and V.E.Z. from 86 localities in the Arkhangelsk oblast during 2009-2013 (Table 1). These samplings took place on: 17.-19.VII.2009; 14.-17.VI. and 7.-9.VIII.2010; 14.-26.VI.2011; 17.-20.VI.2012; 2.-6. and 24.-29.VI.2013. Information was kindly provided also by $\mathrm{M}$. Tähtinen who (together with J. Paukkunen) collected Lepidoptera in SW part of the oblast, in lo- 
Table 1. List of sampling localities in Arkhangelsk oblast.

\begin{tabular}{|c|c|c|c|c|}
\hline \multirow[t]{2}{*}{ Code } & \multirow[t]{2}{*}{ Locality } & \multicolumn{2}{|c|}{ Geographical coordinates* } & \multirow[t]{2}{*}{ No of species** } \\
\hline & & Latitude, N & Longitude, E & \\
\hline ArO & Arkhangelsk oblast & - & - & $6 / 1$ \\
\hline $\mathrm{KaO}$ & Kargopolsky district & - & - & $1 / 0$ \\
\hline Ka1 & Kargopol & $61^{\circ} 30^{\prime} 21^{\prime \prime}$ & $38^{\circ} 56^{\prime} 56^{\prime \prime}$ & $109 / 3$ \\
\hline $\mathrm{Ka} 2$ & Morstchikhinskaya & $61^{\circ} 46^{\prime} 10^{\prime \prime}$ & $38^{\circ} 02^{\prime} 37^{\prime \prime}$ & $10 / 5$ \\
\hline Ka3 & Kenozersky National Park & - & - & $6 / 0$ \\
\hline $\mathrm{Ka} 4$ & 2 km SE Ileksinskaya & $61^{\circ} 40^{\prime} 53^{\prime \prime}$ & $38^{\circ} 05^{\prime} 08^{\prime \prime}$ & $19 / 0$ \\
\hline Ka5 & 7 km E Savinskaya & $61^{\circ} 44^{\prime} 52^{\prime \prime}$ & $39^{\circ} 12^{\prime} 45^{\prime \prime}$ & $17 / 0$ \\
\hline Ka6 & 3 km S Kargopol & $61^{\circ} 29^{\prime} 04^{\prime \prime}$ & $38^{\circ} 57^{\prime} 23^{\prime \prime}$ & $28 / 0$ \\
\hline $\mathrm{Ka} 7$ & lleksinskaya & $61^{\circ} 41^{\prime} 51^{\prime \prime}$ & $38^{\circ} 04^{\prime} 00^{\prime \prime}$ & $39 / 0$ \\
\hline Ka8 & 2 km S Kirillovo & 6132'28" & $39^{\circ} 16^{\prime} 07^{\prime \prime}$ & $29 / 0$ \\
\hline Ka9 & Stegnevskaya & $61^{\circ} 29^{\prime} 26^{\prime \prime}$ & $39^{\circ} 28^{\prime} 53^{\prime \prime}$ & $8 / 0$ \\
\hline Ka10 & 3 km W Zhukovskaya & $61^{\circ} 29^{\prime} 01^{\prime \prime}$ & $38^{\circ} 38^{\prime} 36^{\prime \prime}$ & $33 / 0$ \\
\hline Ka11 & $1 \mathrm{~km} \mathrm{E}$ Khotenovo & $61^{\circ} 12^{\prime} 06^{\prime \prime}$ & $38^{\circ} 35^{\prime} 13^{\prime \prime}$ & $60 / 0$ \\
\hline Ka12 & Kononovo & $61^{\circ} 07^{\prime} 01^{\prime \prime}$ & $38^{\circ} 45^{\prime} 08^{\prime \prime}$ & $33 / 0$ \\
\hline Ka13 & 3 km N Svarozero & $61^{\circ} 02^{\prime} 58^{\prime \prime}$ & $38^{\circ} 32^{\prime} 37^{\prime \prime}$ & $33 / 0$ \\
\hline Ka14 & 1 km NW Dubrovo & $60^{\circ} 58^{\prime} 26^{\prime \prime}$ & $38^{\circ} 32^{\prime} 46^{\prime \prime}$ & $64 / 0$ \\
\hline Ka15 & $6 \mathrm{~km}$ E Stegneevskaya & $61^{\circ} 28^{\prime} 32^{\prime \prime}$ & $39^{\circ} 35^{\prime} 42^{\prime \prime}$ & $41 / 0$ \\
\hline Kb2 & 13 km NW Cherevkovo & $61^{\circ} 53^{\prime} 33^{\prime \prime}$ & $45^{\circ} 09^{\prime} 50^{\prime \prime}$ & $35 / 0$ \\
\hline Kb3 & Cherevkovo & $61^{\circ} 46^{\prime} 52^{\prime \prime}$ & $45^{\circ} 16^{\prime} 08^{\prime \prime}$ & $0 / 2$ \\
\hline Kb4 & 17 km NE Krasnoborsk & $61^{\circ} 37^{\prime} 03^{\prime \prime}$ & $45^{\circ} 39^{\prime} 16^{\prime \prime}$ & $71 / 0$ \\
\hline Kh2 & $5 \mathrm{~km} \mathrm{~W}$ Leunovo & $64^{\circ} 13^{\prime} 20^{\prime \prime}$ & $42^{\circ} 35^{\prime} 45^{\prime \prime}$ & $11 / 0$ \\
\hline Kh3 & Kuzomen & $64^{\circ} 16^{\prime} 17^{\prime \prime}$ & $42^{\circ} 56^{\prime} 32^{\prime \prime}$ & $14 / 0$ \\
\hline Kh4 & 9 km NW Belogorsky & $64^{\circ} 13^{\prime} 29^{\prime \prime}$ & $42^{\circ} 09^{\prime} 52^{\prime \prime}$ & $12 / 0$ \\
\hline Kh5 & 2 km S Ust-Pinega & $64^{\circ} 07^{\prime} 54^{\prime \prime}$ & $41^{\circ} 56^{\prime} 24^{\prime \prime}$ & $8 / 0$ \\
\hline Kh6 & 12 km SE Kholmogory & $64^{\circ} 10^{\prime} 28^{\prime \prime}$ & $41^{\circ} 26^{\prime} 17^{\prime \prime}$ & $26 / 0$ \\
\hline Kh7 & Boloto & $63^{\circ} 38^{\prime}$ & $41^{\circ} 47^{\prime}$ & $11 / 0$ \\
\hline Kh8 & 3 km S Bolshaya Gora & $63^{\circ} 36^{\prime} 06^{\prime \prime}$ & $41^{\circ} 36 ’ 54^{\prime \prime}$ & $31 / 0$ \\
\hline Kh9 & 5 km NE Emetsk & $63^{\circ} 31^{\prime} 00^{\prime \prime}$ & $41^{\circ} 42^{\prime} 54^{\prime \prime}$ & $14 / 0$ \\
\hline Kh10 & $3 \mathrm{~km} \mathrm{~N}$ Oseredok & $63^{\circ} 30^{\prime} 16^{\prime \prime}$ & $41^{\circ} 33^{\prime} 18^{\prime \prime}$ & $16 / 0$ \\
\hline Kh11 & Yemtsa River & - & - & $3 / 0$ \\
\hline Kh12 & 8 km S Emetsk & $63^{\circ} 24^{\prime} 48^{\prime \prime}$ & $41^{\circ} 47^{\prime} 00^{\prime \prime}$ & $20 / 0$ \\
\hline Kh13 & Seltso & $63^{\circ} 19^{\prime}$ & $41^{\circ} 24^{\prime}$ & $2 / 0$ \\
\hline Kh14 & Kurja & $64^{\circ} 16^{\prime}$ & $41^{\circ} 31^{\prime}$ & $1 / 0$ \\
\hline Kh15 & Kurostrov Island & $64^{\circ} 15^{\prime}$ & $41^{\circ} 43^{\prime}$ & $28 / 0$ \\
\hline Kh16 & Naelostrov Island & $64^{\circ} 13^{\prime}$ & $41^{\circ} 46^{\prime}$ & $18 / 0$ \\
\hline Kh17 & Lukovetsky & $64^{\circ} 18^{\prime}$ & $41^{\circ} 55^{\prime}$ & $0 / 1$ \\
\hline Ko1 & Konosha & $60^{\circ} 58^{\prime} 07^{\prime \prime}$ & $40^{\circ} 15^{\prime} 23^{\prime \prime}$ & $0 / 1$ \\
\hline Ko2 & 5 km E Zaruchevskaya & $60^{\circ} 54^{\prime} 02^{\prime \prime}$ & $40^{\circ} 50^{\prime} 38^{\prime \prime}$ & $49 / 0$ \\
\hline Ko3 & Melent'ev Pal & 6111'17"' & $40^{\circ} 18^{\prime} 30^{\prime \prime}$ & $36 / 0$ \\
\hline Kt1 & Kotlas & $61^{\circ} 15^{\prime}$ & $46^{\circ} 40^{\prime}$ & $3 / 283$ \\
\hline $\mathrm{Kt} 2$ & 6 km E Vychegodsky & $61^{\circ} 15^{\prime} 17^{\prime \prime}$ & $46^{\circ} 59^{\prime} 18^{\prime \prime}$ & $10 / 0$ \\
\hline $\mathrm{Kt3}$ & 6 km SW Koryazhma & $61^{\circ} 16^{\prime} 35^{\prime \prime}$ & $47^{\circ} 04^{\prime} 04^{\prime \prime}$ & $15 / 0$ \\
\hline $\mathrm{Kt4}$ & $3 \mathrm{~km}$ E Solvychegodsk & 6119'43" & $46^{\circ} 58^{\prime} 57^{\prime \prime}$ & $36 / 0$ \\
\hline $\mathrm{Kt5}$ & 2 km N Koryazhma & $61^{\circ} 19^{\prime} 53^{\prime \prime}$ & $47^{\circ} 08^{\prime} 42^{\prime \prime}$ & $33 / 0$ \\
\hline Kt6 & Koryazhma & $61^{\circ} 19^{\prime}$ & $47^{\circ} 09^{\prime}$ & $7 / 0$ \\
\hline $\mathrm{Kt} 7$ & $3 \mathrm{~km} \mathrm{~N}$ Zabolotie & $61^{\circ} 23^{\prime} 05^{\prime \prime}$ & $47^{\circ} 11^{\prime} 34^{\prime \prime}$ & $14 / 0$ \\
\hline Kt8 & 1 km W Kharitonovo & $61^{\circ} 24^{\prime} 13^{\prime \prime}$ & $47^{\circ} 28^{\prime} 46^{\prime \prime}$ & $11 / 0$ \\
\hline Le2 & $8 \mathrm{~km} \mathrm{~N}$ Chublashkoe & $64^{\circ} 47^{\prime}$ & $45^{\circ} 11^{\prime}$ & $0 / 3$ \\
\hline Le3 & Vozhgora & $64^{\circ} 33^{\prime}$ & $48^{\circ} 27^{\prime}$ & $0 / 19$ \\
\hline Le4 & Borkovskaya & $65^{\circ} 11^{\prime}$ & $49^{\circ} 34^{\prime}$ & $1 / 0$ \\
\hline Me1 & Mezen & $65^{\circ} 50^{\prime}$ & $44^{\circ} 16^{\prime}$ & $0 / 7$ \\
\hline Me2 & 34 km SE Mezen & $65^{\circ} 34^{\prime} 24^{\prime \prime}$ & $44^{\circ} 37^{\prime} 36^{\prime \prime}$ & $5 / 0$ \\
\hline Me3 & 2 km S Kimzha & $65^{\circ} 33^{\prime} 00^{\prime \prime}$ & $44^{\circ} 36^{\prime} 12^{\prime \prime}$ & $17 / 0$ \\
\hline Me4 & Kamenka & $65^{\circ} 54^{\prime}$ & $44^{\circ} 07^{\prime}$ & $1 / 0$ \\
\hline Me5 & Dolgostchelye & $66^{\circ} 03^{\prime}$ & $43^{\circ} 29^{\prime}$ & $0 / 2$ \\
\hline Me6 & Soyana River & $65^{\circ} 39^{\prime}$ & $42^{\circ} 49^{\prime}$ & $0 / 1$ \\
\hline Ny1 & Nyandoma & $61^{\circ} 38^{\prime} 50^{\prime \prime}$ & $40^{\circ} 09^{\prime} 34^{\prime \prime}$ & $10 / 0$ \\
\hline
\end{tabular}




\begin{tabular}{lll}
\hline Code Locality & \begin{tabular}{c} 
Geographical coordinates* \\
\cline { 2 - 3 }
\end{tabular} & Latitude, $\mathrm{N} \quad$ Longitude, $\mathrm{E}$ \\
\hline
\end{tabular}

\begin{tabular}{|c|c|c|c|c|}
\hline Ono & Onezhsky district & - & - & $8 / 0$ \\
\hline On1 & Onega & $63^{\circ} 55^{\prime}$ & $38^{\circ} 06^{\prime}$ & $3 / 0$ \\
\hline On2 & $7 \mathrm{~km}$ N Pokrovskoye & $64^{\circ} 04^{\prime} 35^{\prime \prime}$ & $38^{\circ} 03^{\prime} 14^{\prime \prime}$ & $3 / 0$ \\
\hline On3 & $1 \mathrm{~km}$ N Pokrovskoye & $64^{\circ} 01^{\prime} 20^{\prime \prime}$ & $38^{\circ} 05^{\prime} 52^{\prime \prime}$ & $25 / 0$ \\
\hline On4 & $3 \mathrm{~km}$ SE Anda-Kirpichnaya & $63^{\circ} 50^{\prime} 18^{\prime \prime}$ & $38^{\circ} 16^{\prime} 17^{\prime \prime}$ & $19 / 0$ \\
\hline On5 & Maloschuika & $63^{\circ} 42^{\prime} 57^{\prime \prime}$ & $37^{\circ} 27^{\prime} 11^{\prime \prime}$ & $3 / 0$ \\
\hline On6 & $2 \mathrm{~km}$ NE Pavlovsky Bor & $63^{\circ} 36^{\prime} 26^{\prime \prime}$ & $39^{\circ} 05^{\prime} 19^{\prime \prime}$ & $29 / 0$ \\
\hline On7 & 4 km NW Kopylovka & $63^{\circ} 00^{\prime} 38^{\prime \prime}$ & $39^{\circ} 17^{\prime} 42^{\prime \prime}$ & $11 / 0$ \\
\hline On8 & Kamenikha & $63^{\circ} 49^{\prime}$ & $38^{\circ} 20^{\prime}$ & $0 / 1$ \\
\hline $\mathrm{PiO}$ & Pinezhsky district & - & - & $0 / 2$ \\
\hline $\mathrm{Pi} 2$ & 9 km S Kuloy & $64^{\circ} 54^{\prime} 18^{\prime \prime}$ & $43^{\circ} 31^{\prime} 06^{\prime \prime}$ & $25 / 0$ \\
\hline $\mathrm{Pi} 3$ & Pinega & $64^{\circ} 42^{\prime} 19^{\prime \prime}$ & $43^{\circ} 23^{\prime} 44^{\prime \prime}$ & $8 / 9$ \\
\hline $\mathrm{Pi} 4$ & Golubino & $64^{\circ} 33^{\prime}$ & $43^{\circ} 16^{\prime}$ & $67 / 146$ \\
\hline $\mathrm{Pi} 5$ & Kokornaya & $64^{\circ} 33^{\prime}$ & $44^{\circ} 37^{\prime}$ & $0 / 2$ \\
\hline Pi6 & $6 \mathrm{~km} \mathrm{SW}$ Soyala & $64^{\circ} 27^{\prime} 08^{\prime \prime}$ & $43^{\circ} 10^{\prime} 20^{\prime \prime}$ & $22 / 0$ \\
\hline $\mathrm{Pi} 7$ & Polta River, lower flow & $64^{\circ} 59^{\prime}$ & $43^{\circ} 08^{\prime}$ & $0 / 2$ \\
\hline $\mathrm{Pi} 8$ & Nyukhcha & $63^{\circ} 26^{\prime}$ & $46^{\circ} 32^{\prime}$ & $0 / 1$ \\
\hline Pi9 & Yula River, $50 \mathrm{~km}$ above Kushkopala & $63^{\circ} 29^{\prime}$ & $44^{\circ} 17^{\prime}$ & $0 / 1$ \\
\hline $\mathrm{Pi} 10$ & Ust-Ezhuga & $64^{\circ} 28^{\prime}$ & $44^{\circ} 16^{\prime}$ & $2 / 0$ \\
\hline $\mathrm{PIO}$ & Plesetsky district & _ & - & $4 / 0$ \\
\hline PI1 & Plesetsk & $62^{\circ} 43^{\prime}$ & $40^{\circ} 17^{\prime}$ & $1 / 0$ \\
\hline $\mathrm{PI} 2$ & Lomovoe & $64^{\circ} 01^{\prime}$ & $40^{\circ} 39^{\prime}$ & $124 / 29$ \\
\hline $\mathrm{PI} 3$ & Kholmogorskaya & $63^{\circ} 48^{\prime}$ & $40^{\circ} 39^{\prime}$ & $119 / 6$ \\
\hline $\mathrm{PI} 4$ & $6 \mathrm{~km}$ NE Yarnema & $62^{\circ} 59^{\prime} 06^{\prime \prime}$ & $39^{\circ} 28^{\prime} 30^{\prime \prime}$ & $26 / 0$ \\
\hline PI5 & $6 \mathrm{~km} \mathrm{~S} \mathrm{Sheleksa}$ & $62^{\circ} 50^{\prime} 21^{\prime \prime}$ & $40^{\circ} 17^{\prime} 19^{\prime \prime}$ & $5 / 0$ \\
\hline PI6 & Mirnyi & $62^{\circ} 46^{\prime}$ & $40^{\circ} 20^{\prime}$ & $13 / 0$ \\
\hline $\mathrm{PI} 7$ & $4 \mathrm{~km}$ SE Plesetsk & $62^{\circ} 41^{\prime} 49^{\prime \prime}$ & $40^{\circ} 13^{\prime} 42^{\prime \prime}$ & $34 / 0$ \\
\hline $\mathrm{PI} 8$ & $3 \mathrm{~km} \mathrm{~S} \mathrm{Lipakovo}$ & $62^{\circ} 22^{\prime} 48^{\prime \prime}$ & $39^{\circ} 40^{\prime} 58^{\prime \prime}$ & $17 / 0$ \\
\hline PI9 & Fedosovo & $62^{\circ} 07^{\prime}$ & $38^{\circ} 09^{\prime}$ & $3 / 0$ \\
\hline PI10 & $7 \mathrm{~km} \mathrm{~S} \mathrm{Konevo}$ & $62^{\circ} 03^{\prime} 36^{\prime \prime}$ & $39^{\circ} 15^{\prime} 00^{\prime \prime}$ & $23 / 0$ \\
\hline PrO & Primorsky district & - & - & $5 / 5$ \\
\hline Pr1 & Arkhangelsk & $64^{\circ} 33^{\prime}$ & $40^{\circ} 33^{\prime}$ & $233 / 43$ \\
\hline Pr2 & Mudyugsky lighthouse & $64^{\circ} 55^{\prime}$ & $40^{\circ} 14^{\prime}$ & $24 / 0$ \\
\hline Pr3 & Lapominka & $64^{\circ} 47^{\prime} 09^{\prime \prime}$ & $40^{\circ} 28^{\prime} 02^{\prime \prime}$ & $11 / 2$ \\
\hline Pr4 & Neftebaza & $64^{\circ} 39^{\prime}$ & $40^{\circ} 41^{\prime}$ & $3 / 0$ \\
\hline Pr5 & Talagi & $64^{\circ} 38^{\prime}$ & $40^{\circ} 38^{\prime}$ & $9 / 4$ \\
\hline Pr6 & Molodezhnyi Island & $64^{\circ} 34^{\prime}$ & $40^{\circ} 29^{\prime}$ & $9 / 0$ \\
\hline Pr7 & Kegostrov & $64^{\circ} 33^{\prime}$ & $40^{\circ} 25^{\prime}$ & $0 / 1$ \\
\hline Pr8 & IIma & $64^{\circ} 32^{\prime}$ & $40^{\circ} 42^{\prime}$ & $3 / 0$ \\
\hline Pr9 & Krasnoflotsky Island & $64^{\circ} 29^{\prime} 22^{\prime \prime}$ & $40^{\circ} 39^{\prime} 14^{\prime \prime}$ & $39 / 4$ \\
\hline Pr10 & $60 \mathrm{~km}$ W Severodvinsk & $64^{\circ} 29^{\prime} 30^{\prime \prime}$ & $38^{\circ} 37^{\prime} 48^{\prime \prime}$ & $22 / 0$ \\
\hline Pr11 & Yagry Island & $64^{\circ} 37^{\prime} 52^{\prime \prime}$ & $39^{\circ} 49^{\prime} 59^{\prime \prime}$ & $14 / 0$ \\
\hline Pr12 & Severodvinsk & $64^{\circ} 35^{\prime} 33^{\prime \prime}$ & $39^{\circ} 50^{\prime} 58^{\prime \prime}$ & $35 / 2$ \\
\hline Pr13 & Yuras & $64^{\circ} 31^{\prime} 06^{\prime \prime}$ & $40^{\circ} 41^{\prime} 22^{\prime \prime}$ & $8 / 4$ \\
\hline Pr14 & Uemsky & $64^{\circ} 29^{\prime}$ & $40^{\circ} 59^{\prime}$ & $3 / 0$ \\
\hline Pr15 & Zaostrovje & $64^{\circ} 28^{\prime}$ & $40^{\circ} 30^{\prime}$ & $5 / 0$ \\
\hline Pr16 & Malye Karely & $64^{\circ} 27^{\prime}$ & $40^{\circ} 58^{\prime}$ & $331 / 54$ \\
\hline Pr17 & Babonegovo & $64^{\circ} 26^{\prime}$ & $40^{\circ} 58^{\prime}$ & $17 / 10$ \\
\hline Pr18 & Psarevo & $64^{\circ} 26^{\prime}$ & $41^{\circ} 00^{\prime}$ & $3 / 2$ \\
\hline Pr19 & Lodma & $64^{\circ} 25^{\prime}$ & $41^{\circ} 18^{\prime}$ & $0 / 5$ \\
\hline Pr20 & Katunino & $64^{\circ} 24^{\prime}$ & $40^{\circ} 37^{\prime}$ & $2 / 0$ \\
\hline Pr21 & Khorkovo, Lyavlya & $64^{\circ} 23^{\prime}$ & $41^{\circ} 01^{\prime}$ & $13 / 2$ \\
\hline Pr22 & Lesnaya Rechka & $64^{\circ} 25^{\prime}$ & $40^{\circ} 38^{\prime}$ & $0 / 1$ \\
\hline Pr23 & Vologodskoe shosse & $64^{\circ} 21^{\prime}$ & $40^{\circ} 58^{\prime}$ & $9 / 6$ \\
\hline Pr24 & Chasovenskoe & $64^{\circ} 28^{\prime}$ & $40^{\circ} 38^{\prime}$ & $16 / 0$ \\
\hline Pr25 & Bobrovo & $64^{\circ} 21^{\prime}$ & $41^{\circ} 10^{\prime}$ & $3 / 0$ \\
\hline Pr26 & Nenoksa & $64^{\circ} 37^{\prime}$ & $39^{\circ} 12^{\prime}$ & $5 / 1$ \\
\hline Pr27 & Mechka & $64^{\circ} 23^{\prime}$ & $40^{\circ} 51^{\prime}$ & $5 / 0$ \\
\hline
\end{tabular}




\begin{tabular}{lll}
\hline Code Locality & $\frac{\text { Geographical coordinates }^{*}}{\text { Latitude, } N \quad \text { Longitude, } \mathrm{E}}$ \\
\hline
\end{tabular}

\begin{tabular}{|c|c|c|c|c|}
\hline Pr28 & Laisky Dok & $64^{\circ} 32^{\prime}$ & $40^{\circ} 15^{\prime}$ & $13 / 0$ \\
\hline Pr29 & Zolotitsa River, upper flow & $65^{\circ} 39^{\prime}$ & $41^{\circ} 06^{\prime}$ & $0 / 1$ \\
\hline Pr30 & Halt 25th km & $64^{\circ} 29^{\prime}$ & $41^{\circ} 02^{\prime}$ & $0 / 1$ \\
\hline Pr31 & Halt 36th km & $64^{\circ} 27^{\prime}$ & $41^{\circ} 14^{\prime}$ & $0 / 1$ \\
\hline Pr32 & Izhma & $64^{\circ} 46^{\prime}$ & $40^{\circ} 47^{\prime}$ & $0 / 8$ \\
\hline Pr33 & Ilmatikha River, upper flow & $64^{\circ} 33^{\prime}$ & $40^{\circ} 43^{\prime}$ & $0 / 1$ \\
\hline Pr34 & Chidvia River, upper flow & $64^{\circ} 54^{\prime}$ & $41^{\circ} 02^{\prime}$ & $0 / 1$ \\
\hline Pr35 & Mouth of Ivovik River & $65^{\circ} 27^{\prime}$ & $39^{\circ} 43^{\prime}$ & $0 / 1$ \\
\hline Sh1 & Shenkursk & $62^{\circ} 06^{\prime} 25^{\prime \prime}$ & $42^{\circ} 54^{\prime} 36^{\prime \prime}$ & $2 / 1$ \\
\hline Sh2 & 5 km S Ust-Padenga & $61^{\circ} 51^{\prime} 49^{\prime \prime}$ & $42^{\circ} 38^{\prime} 01^{\prime \prime}$ & $43 / 0$ \\
\hline Sh3 & 2 km W Rovdino & $61^{\circ} 40^{\prime} 41^{\prime \prime}$ & $42^{\circ} 30^{\prime} 02^{\prime \prime}$ & $4 / 0$ \\
\hline Sh4 & Rudinskaya & $61^{\circ} 46^{\prime} 02^{\prime \prime}$ & $42^{\circ} 34^{\prime} 57^{\prime \prime}$ & $93 / 0$ \\
\hline SoO & Solovetskie Islands & - & - & 6/35 \\
\hline So1 & Bolshoi Solovetsky Is. & - & - & $55 / 1$ \\
\hline So2 & Anzer Is. & $65^{\circ} 09^{\prime}$ & $36^{\circ} 03^{\prime}$ & $2 / 0$ \\
\hline So3 & Bolshoi Zayatsky Is. & $64^{\circ} 58^{\prime}$ & $35^{\circ} 40^{\prime}$ & $2 / 0$ \\
\hline So4 & Bolshaya Muksalma Is. & $65^{\circ} 02^{\prime}$ & $35^{\circ} 57^{\prime}$ & $50 / 0$ \\
\hline So5 & Botanical Garden & $65^{\circ} 03^{\prime} 09^{\prime \prime}$ & $35^{\circ} 39^{\prime} 50^{\prime \prime}$ & $30 / 0$ \\
\hline So6 & Near Solovetsky settlement & $65^{\circ} 01^{\prime}$ & $35^{\circ} 45^{\prime}$ & $109 / 0$ \\
\hline So7 & Pechak Cape & $64^{\circ} 57^{\prime}$ & $35^{\circ} 45^{\prime}$ & $43 / 0$ \\
\hline So8 & So1 near the dam leading to So 4 & $65^{\circ} 01^{\prime} 11^{\prime \prime}$ & $35^{\circ} 52^{\prime} 24^{\prime \prime}$ & $16 / 0$ \\
\hline So9 & Malaya Muksalma Is. & - & - & $2 / 0$ \\
\hline Us1 & Oktyabrsky & $61^{\circ} 05^{\prime}$ & $43^{\circ} 10^{\prime}$ & $1 / 0$ \\
\hline Us2 & 10 km E Kizema & $61^{\circ} 08^{\prime}$ & $44^{\circ} 59^{\prime}$ & $18 / 0$ \\
\hline Us3 & 7 km NWW Oktyabrsky & $61^{\circ} 09^{\prime} 04^{\prime \prime}$ & $43^{\circ} 05^{\prime} 43^{\prime \prime}$ & $46 / 0$ \\
\hline Us4 & 6 km NWW Oktyabrsky & $61^{\circ} 06^{\prime} 36^{\prime \prime}$ & $43^{\circ} 03^{\prime} 53^{\prime \prime}$ & $60 / 0$ \\
\hline Us5 & 4 km S Oktyabrsky & $61^{\circ} 02^{\prime} 05^{\prime \prime}$ & $43^{\circ} 13^{\prime} 52^{\prime \prime}$ & $28 / 0$ \\
\hline Us6 & 6 km N Uglovskaya & $60^{\circ} 58^{\prime} 01^{\prime \prime}$ & $43^{\circ} 12^{\prime} 38^{\prime \prime}$ & $29 / 0$ \\
\hline Ve1 & Velsk & $61^{\circ} 04^{\prime}$ & $42^{\circ} 06^{\prime}$ & $87 / 0$ \\
\hline Ve2 & 5 km N Priluki & $61^{\circ} 21^{\prime} 22^{\prime \prime}$ & $42^{\circ} 23^{\prime} 01^{\prime \prime}$ & $25 / 0$ \\
\hline Ve3 & 12 km NE Velsk & $61^{\circ} 09^{\prime} 26^{\prime \prime}$ & $42^{\circ} 12^{\prime} 39^{\prime \prime}$ & $21 / 0$ \\
\hline Ve4 & 9 km NE Velsk & $61^{\circ} 08^{\prime} 05^{\prime \prime}$ & $42^{\circ} 11^{\prime} 27^{\prime \prime}$ & $42 / 0$ \\
\hline Ve5 & 7 km NE Velsk & $61^{\circ} 07^{\prime} 15^{\prime \prime}$ & $42^{\circ} 10^{\prime} 36^{\prime \prime}$ & $33 / 0$ \\
\hline Ve6 & $12 \mathrm{~km}$ SE Velsk & $61^{\circ} 01^{\prime}$ & $42^{\circ} 20^{\prime}$ & $16 / 0$ \\
\hline Ve7 & 7 km E Kozlovskaya & $61^{\circ} 05^{\prime} 35^{\prime \prime}$ & $42^{\circ} 58^{\prime} 05^{\prime \prime}$ & $21 / 0$ \\
\hline Ve8 & 1 km W Priluk & $61^{\circ} 00^{\prime} 00^{\prime \prime}$ & $42^{\circ} 24^{\prime} 23^{\prime \prime}$ & $42 / 0$ \\
\hline Ve9 & 7 km W Verkhnepuisky & $61^{\circ} 36^{\prime} 56^{\prime \prime}$ & $41^{\circ} 16^{\prime} 14^{\prime \prime}$ & $21 / 0$ \\
\hline Vg1 & Vilegodsk & $61^{\circ} 10^{\prime}$ & $48^{\circ} 17^{\prime}$ & $11 / 0$ \\
\hline Vi1 & Bereznik & $62^{\circ} 50^{\prime} 46^{\prime \prime}$ & $42^{\circ} 44^{\prime} 02^{\prime \prime}$ & $1 / 0$ \\
\hline Vi2 & 1 km S Monastyrek & $63^{\circ} 03^{\prime} 46^{\prime \prime}$ & $42^{\circ} 04^{\prime} 11^{\prime \prime}$ & $36 / 0$ \\
\hline Vi3 & Uyta & $63^{\circ} 00^{\prime} 36^{\prime \prime}$ & $42^{\circ} 31^{\prime} 36^{\prime \prime}$ & $142 / 0$ \\
\hline Vi4 & Ust-Vaenga & $63^{\circ} 00^{\prime}$ & $42^{\circ} 38^{\prime}$ & $4 / 0$ \\
\hline Vi5 & 2 km SE Bereznik & $62^{\circ} 49^{\prime} 54^{\prime \prime}$ & $42^{\circ} 46^{\prime} 15^{\prime \prime}$ & $37 / 0$ \\
\hline Vi6 & 6 km S Zaborye & $62^{\circ} 41^{\prime} 52^{\prime \prime}$ & $42^{\circ} 53^{\prime} 35^{\prime \prime}$ & $31 / 0$ \\
\hline Vi7 & 6 km S Rochegda & $62^{\circ} 37^{\prime} 21^{\prime \prime}$ & $43^{\circ} 27^{\prime} 24^{\prime \prime}$ & $25 / 0$ \\
\hline Vi8 & Tulgas & $62^{\circ} 35^{\prime} 32^{\prime \prime}$ & $43^{\circ} 30^{\prime} 35^{\prime \prime}$ & $40 / 0$ \\
\hline Vi9 & Ura (abandoned) & $63^{\circ} 00^{\prime}$ & $45^{\circ} 20^{\prime}$ & $0 / 1$ \\
\hline Vi10 & Shivrey River & $62^{\circ} 54^{\prime}$ & $44^{\circ} 31^{\prime}$ & $0 / 2$ \\
\hline Vt2 & 50 km S Mamonikha & $63^{\circ} 00^{\prime} 43^{\prime \prime}$ & $45^{\circ} 39^{\prime} 37^{\prime \prime}$ & $0 / 10$ \\
\hline Vt3 & 2 km W Osiyevskaya & $62^{\circ} 20^{\prime} 24^{\prime \prime}$ & $44^{\circ} 06^{\prime} 56^{\prime \prime}$ & $42 / 0$ \\
\hline Vt4 & 3 km W Verkhnyaya Toima & $62^{\circ} 13^{\prime} 46^{\prime \prime}$ & $44^{\circ} 57^{\prime} 04^{\prime \prime}$ & $19 / 0$ \\
\hline Vt5 & 12 km S Verkhnyaya Toima & $62^{\circ} 08^{\prime} 15^{\prime \prime}$ & $45^{\circ} 04^{\prime} 03^{\prime \prime}$ & $18 / 0$ \\
\hline Vt6 & 10 km SW Osiyevskaya & $62^{\circ} 18^{\prime} 03^{\prime \prime}$ & $44^{\circ} 19^{\prime} 12^{\prime \prime}$ & $38 / 0$ \\
\hline
\end{tabular}

*Geographical coordinates were rounded off to minutes when the sampled area exceeded $1 \mathrm{~km}^{2}$ or when the extent of the historical sampling area cannot be recovered. Coordinates are not provided when the locality name refers to an area exceeding $100 \mathrm{~km}^{2}$. **Based on examined specimens / published records. Data for large territories (entire Arkhangelsk oblast or one of administrative units within it) are included only for species that have not been reported from any clearly specified locality within this territory. 
calities Ka15, Sh4 and Ve9 (Table 1), on 12.VI. 21.VII.2013. The specimens were mostly collected by netting and the total collecting time was ca. 210 person-hours. We performed short-term (1-5 nights) light trapping in four localities: Ka1 on 25.-26.VII. 2011 and 18.-20.VI.2012, Pi4 on 21.-22.VII.2011, So6 on 24.-29.VI.2013, and Ve1 on 16.-18.VII.2012. In addition, we arranged a 10 week-long light trapping in one locality, Vi3, on 15.VII. - 28. IX.2011. We also recorded easily identifiable species on the basis of visual observations, collected leaf mines and reared moths from field-collected larvae.

The materials are mostly deposited in the Zoological Museum, University of Helsinki $(\mathrm{MZH})$. Some specimens were donated to the Zoological Museum in St. Petersburg (ZISP), and samples of 2011 were partially donated to the Natural History Museum in London (NHM).

Additional information was obtained by studying collections of ZISP, where we checked materials of each of 717 species that were reported (Sinev 2008) from the biogeographical region that includes Arkhangelsk oblast. We also sought for specimens from Arkhangelsk oblast in so-called "Arctic collection" created by N. Y. Kusnezov in 1930s and in accession materials. In the Northern (Arctic) Federal University (NarFU), Arkhangelsk, we checked, sorted and identified all specimens (some 2,000 exx) collected by L. F. Zelenova; most of this material is now transferred to ZISP. We also studied reference collections of NarFU, samples collected by students during the summer courses and photographic images from the private collection of Anatoly Popov, Arkhangelsk.

In the following list, an asterisk $(*)$ denotes species that are reported from Arkhangelsk oblast for the first time. The references to earlier publications are given only if there are no specimens examined by the authors. For each species, we provide the list of localities (Table 1, Fig. 2). Codes of the localities include two-letter abbreviations of the administrative units ("rayon", translated as district) within the Arkhangelsk oblast (Fig. 1) and a numerical code, with 0 referring to a record made from this administrative unit which cannot be attributed to any specific locality within it, and with 1 referring to the administrative centre of respective unit. The only exception is
Solovetsky Archipelago, for which we used the codes starting with "So", although this archipelago administratively belongs to Primorsky district. A dash between the numerical codes indicates that the species was recorded in all localities whose numbers lie between those connected by a dash. The order of families follows Nieukerken et al. (2011), the order of genera and species within a family mostly follows Sinev (2008), whereas species-level nomenclature is given after Fauna Europaea (Karsholt et al. 2013).

When commenting distribution records, we used all available sources of information, primarily Kullberg et al. (2014), Hyönteistietokanta (2014) and Sinev (2008). The references to the regions of Russia follow the subdivision of the country adopted by Sinev (2008). Biogeographical provinces of Finland are given in italics; for a map of the provinces, consult Kullberg et al. (2002) or Haarto and Winqvist (2006).

\section{List of species}

Micropterigidae

* Micropterix aruncella (Scop.). Ve1.

*M. aureatella (Scop.). Kh8, Kh12, Kt3, P12, P17, Pr16, So6, Us3, Vi2, Vi7-8, Vt6.

*M. calthella (L.). Ka11, Kb4, Kh8, Ko3, P17, Pr9, Ve1, Ve4, Vi2, Vi7, Vt3, Vt6.

*M. mansuetella Z. Ka11.

Eriocraniidae

*Eriocrania cicatricella (Zett.). Pr16.

*E. semipurpurella (Steph.). Pr16.

*E. sparrmannella (Bosc). Kh9, Kt3, Pi2, Sh2, Ve4, Vi6.

Hepialidae

Gazoryctra ganna (Hbn.). Pi4 (A. Tikhomirov, pers. comm.).

Pharmacis fusconebulosa (De Geer). Pi4 (A. Tikhomirov, pers. comm.).

Phymatopus hecta (L.). Kb4, Kh6, Kh10, Kh15, Kt8, Pi4, P13, Pr16, Sh2, So1, Vi2.

Hepialus humuli (L.). Kh15, Kt1, Pr16-17.

Nepticulidae

* Stigmella anomalella (Goeze). Pi4, Pr1.

*S. assimilella (Z.). Pr1. 
*S. betulicola (Stt.). Kh9, Pi2, Sh2, Vi6.

*S. confusella (Wood \& Wals.). Sh2, Vi6.

*S. lapponica (Wck.). Ka6, Kh2, Kh4, Kh9, On6, Pi2, Pr1, Sh2, Ve4, Vi6.

$*$ S. lemniscella (Z.). Ve1.

*S. luteella (Stt.). Pr1.

*S. magdalenae (Klim.). Ka4, Kh6, Kt4, On4, On5, Pr1, Sh2, Ve3, Vi7.

*S. nylandriella (Tengstr.). Pr1.

*S. sorbi (Stt.). Ka4, Ka11, Ka13, Ka14, Kt4, Pi4,

Pr1, Us4, Ve1, Ve3-4, Vi5, Vt3.

*S. splendidissimella (H.-S.). Ka6, Pi4.

*S. trimaculella (Haw.). Kt1, Kt6, P11, Pr1, Sh1, Vel.

*Bohemannia pulverosella (Stt.). Kt1.

*Ectoedemia intimella (Z.). Ka14.

*E. minimella (Zett.). Kt3.

*E. occultella (L.). Kt3.

Opostegidae

*Opostega salaciella (Tr.). Vi2, Vi5.

Heliozelidae

*Heliozela hammoniella Sorh. Ka14.

Adelidae

*Nemophora amatella (Stgr.). P12-3, Pr16.

N. degeerella (L.). Kb4, Kt1, P110, Vi7.

*Adela croesella (Scop.). Ka7.

*A. cuprella (Den. \& Schiff.). Pr16.

* Cauchas fibulella (Den. \& Schiff.). Ka7, Ka1314, Kt3, Us4, Ve1, Ve7-8.

Nematopogon pilella (Den. \& Schiff.). Kt1

(Krulikovsky 1909).

N. robertella (Cl.). Kt1, P13, So6.

$* N$. schwarziellus Z. So1.

Incurvariidae

Alloclemensia mesospilella (H.-S.). Pr1, Pr16

(Zelenova 1972).

Incurvaria oehlmaniella (Hbn.). Me3, Pl2-3, Pr16, So6-7.

I. pectinea Haw. Kt1, Pi2, Pr16, Pr23, So6, So7, Vi6.

I. praelatella (Den. \& Schiff.). P12.

*Phylloporia bistrigella (Haw.). Pr16, Sh2.

Prodoxidae

Lampronia capitella (Cl.). Ka11, P12-3, Pr1, Pr16, Ve1.
L. corticella (L.). Kt1, Pr16.

L. fuscatella (Tengstr.). Pr16 (Zelenova 1972).

*L. luzella (Hbn.). So6.

*L. provectella (Heyd.). Pr16. This is the northernmost record of this rarely collected boreomontane Euro-Siberian species which has not been discovered in the Nordic countries yet. The nearest and the only Fennoscandian record is from Russian Karelia: the single female, collected 17.VII.1863 in humid semi-open spruce forest at "Käpselgä" (Käppäselkä, 6240'04" N, 34¹6'16”'E), was described as Lampronia triangulifera by Tengström (1869).

* L. redimitella (Lienig \& Z.). Pr1.

*L. rupella (Den. \& Schiff.). Pi4, P12-3, Pr16, Us4.

*L. standfussiella Z. P13. This is a rare species which was for a long time known from Fennoscandia only by a specimen (deposited in $\mathrm{MZH}$ ) that was collected near Petrozavodsk in Russian Karelia by A. Günther. Recently it has been reported from northern Finland (Itämies et al. 1996), Kola Peninsula (Kozlov \& Kullberg 2006) and Sweden (Svensson 2005). It has also been collected from Labytnangi on the Asian side of the Ural Mts. (J. K., pers. obs.).

Tischeriidae

*Tischeria angusticollella (Dup.). Ka4, Kh8, Kt5, Pi4, P14, P17, Pr1, Pr10, Ve5, Vi7.

Psychidae

* Siederia rupicolella (Sauter). P13.

Taleporia tubulosa (Retz.). Kt1, P13, Pr16, So6. Psyche casta (Pallas). Kt1 (Krulikovsky 1909).

* Psyche crassiorella (Bruand). Ka1, Ko3, Vi2.

* Acanthopsyche atra (L.). Ka7.

Canephora hirsuta (Poda). Kt1 (Krulikovsky 1909).

*Sterrhopterix standfussi (Wck.). Kh10, Ko2.

Tineidae

Haplotinea insectella (F.). Kt1, Pr1, Ve1.

Agnathosia mendicella (Den. \& Schiff.). Pr1.

Montescardia tessulatella (Lienig \& Z.). Kt1, Pr1, Pr16, So6, Ve1.

Scardia boletella (F.). Kt1 (Krulikovsky 1909).

*Triaxomera fulvimitrella (Sodoffsky). So6. 
Archinemapogon yildizae Koçak. Kt1, Pl2.

Nemapogon cloacella (Haw.). Kt1, Pi4, P12, Pr1, Pr16, So6.

N. granella (L.). Kt1 (Krulikovsky 1909).

*N. wolffiella Karsholt \& Nielsen. Pr16.

Tineola bisseliella (Hummel). Kt1, Pr1.

Tinea pellionella L. Ka1, Kt1, P12, Pr1, Pr16, Vi3.

* Niditinea fuscella (L.). Pr1, Pr16.

*N. truncicolella (Tengstr.). So6.

Monopis laevigella (Den. \& Schiff.). Kt1, Pr1, Pr16, So6.

M. spilotella (Tengstr.). Kt1, Pr16.

*M. weaverella (Scott). Ka11.

Bucculatricidae

*Bucculatrix cidarella (Z.). Ka4, Ka7, Pr1.

*B. cristatella (Z.). Ka12, Kt3, So6.

*B. demaryella (Dup.). Kh2, Sh2, Ve4, Vi6.

*B. frangutella (Goeze). Ka11.

*B. maritima Stt. So6.

*B. nigricomella (Z.). Ka14, Pr16, Sh2.

Gracillariidae

* Gracillaria syringella (F.). Pr1.

*Caloptilia betulicola (M. Hering). Kh9, Pi2, P13, Vi6.

C. elongella (L.). Kt1, Pr1, Pr16.

C. stigmatella (F.). Kt1, Pi4, P13, Pr1, Pr7, Pr16. ${ }^{*}$ C. suberinella (Tengstr.). Kb4, Kh2, Kh6, Kh9, On4, Pi2, Pi4, Pi6, P12, Ve4, Vi2, Vi5, Vi8.

*Euspilapteryx auroguttella (Steph.). P12, P110.

* Parornix anglicella (Stt.). Kt6, P13.

*P. devoniella (Stt.). Pr1, Pr24.

*P. loganella (Stt.). Pr1, Pr16.

*Phyllonorycter apparella (H.-S.). Kt4-5, Pr1, Vt5.

P. cavella (Z.). Pr1, Pr16.

*P. issikii (Kumata). Kt6. This species originates from the Russian Far East and Japan, but has recently aggressively expanded in Europe including Baltic countries and Finland (Karsholt et al. 2013). This record (made on planted limes outside their natural range) most likely lies at the current northern distribution limit, because the extensive search of mines on limes in more northern localities in 20112013 yielded no positive results.

P. sorbi (Frey). Ka4, Kt1, Pr1, Ve3.

*P. strigulatella (Lienig \& Z.). Ka6-7, Ka11, Ka14, Ko3, Pr1, Pr9, Pr16, Ve7, Vi2.
*P. ulmifoliella (Hbn.). Pr16, Us3, Us4.

*P. salicicolella (Sircom). Pr16.

*P. salictella (Z.). Pr16.

P. emberizaepennella (Bouché). Pr1, Pr16.

Phyllocnistis labyrinthella (Bjerk.). Ka1, Ka7, On2, On6, Pr1, Pr16, Vi5.

*P. unipunctella (Steph.). Pr1. On cultivated Populus spp.

Yponomeutidae

Yponomeuta evonymella (L.). Kb3, Kt1, Pr1, Ve1, Vi3.

*Y. sedella (Retz.). Ka1, Vi3.

*Euhyponomeutoides albithoracellus Gaj. Pr1, Pr16.

Swammerdamia caesiella (Hbn.). P13, Pr1, Pr16, $\operatorname{Pr} 23$.

${ }^{*}$ S. compunctella H.-S. P12, Pr16.

*S. passerella (Zett.). Pr16.

* Paraswammerdamia conspersella (Tengstr.). P13, Pr1, Pr16.

*P. lapponica (W. Petersen). Pr16.

Cedestis gysseleniella Z. P12, Pr16.

*Atemelia torquatella (Lienig \& Z.). Pr1.

Argyresthiidae

*Argyresthia dilectella Z. Pi4.

*A. glabratella (Z.). Kh9, So5-6.

A. laevigatella (Heyd.). Pr16.

A. praecocella Z. Pr16.

A. brockeella (Hbn.). Pr1.

A. conjugella Z. Kb2, Kh6, $\mathrm{P} 12, \operatorname{Pr} 1, \operatorname{Pr} 16, \operatorname{Pr} 24$, So4-5, Vi2, Vi8, Vt3.

*A. pygmaeella (Den. \& Schiff.). Pr1, Pr9, Pr16, So5.

A. retinella Z. Pr1, Pr16 (Zelenova 1972).

A. sorbiella (Tr.). P13, Pr1, Pr16.

Plutellidae

Plutella xylostella (L.). Ka1, Ka6, Kh12, Ko1-2, Kt1-2, Me3, Pi4, P12, P110, Pr1, Pr4, Pr16, Sh2, So4-6, Ve1, Ve7, Vi3, Vi5-6, Vi8.

*P. porrectella (L.). Ka1.

Glyphipterigidae

* Digitivalva reticulella (Hbn.). Pi4, P12, Vi5.

Glyphipterix forsterella (F.). Ka13, So7.

*G. haworthana (Steph.). Me3, So4.

*G. simpliciella (Steph.). Ka7, Ka10-12, Ka14, Kh12, Kt2-3, Ko3, Pi3, Pr1, Sh2, So6, Us4, 
Us6, Ve1, Ve4, Ve8, Vi6.

*G. thrasonella (Scop.). Pi4, P12, P17, Sh2, Ve2, Vi2, Vt6.

Ypsolophidae

Ypsolopha dentella (F.). Pr1, Pr16, Pr23.

*Y. falcella (Den. \& Schiff.). Kt6, Pr1.

*Y. nemorella (L.). Pr16.

Y. parenthesella (L.). P13, Pr1, So6, Vi3.

* Ochsenheimeria urella F. v. R. P110.

Lyonetiidae

*Leucoptera sinuella (Reutti). Ka6, Kb2, Kt8.

*L. malifoliella (O. Costa). Kt1, Kt5-7, Ve1.

*Lyonetia clerkella (L.). Kh10, Pr1, Vi5.

*L. ledi Wck. On3.

Douglasiidae

*Tinagma perdicella Z. Ka14.

Oecophoridae

*Denisia similella (Hbn.). Ka1, P12, Pr16, So6.

*D. stipella (L.). P12-3, So6.

*Borkhausenia fuscescens (Haw.). Vi8.

*B. luridicomella (H.-S.). Ka1.

Endrosis sarcitrella (L.). Kt1, P12, Pr1, Pr16.

* Pleurota bicostella (Cl.). Ve2.

* Pseudatemelia josephinae (Toll). P12.

Elachistidae

Semioscopis avellanella (Hbn.). Pr16.

*S. steinkellneriana (Den. \& Schiff.). Pr16.

S. strigulana (F.). Pr16.

Levipalpus hepatariella (Lienig \& Z.). Kt1 (Krulikovsky 1909).

* Exaeretia allisella Stt. Vi3.

E. ciniflonella (Lienig \& Z.). Kt1, P12, So0.

* Agonopterix angelicella (Hbn.). Pi4, Pr1, Pr16.

*A. conterminella (Z.). Pr1, Pr6, Pr16.

A. heracliana (L.). Kt1, Pr16.

*A. hypericella (Hbn.). Pi4.

A. kaekeritziana (L.). Kt1 (Krulikovsky 1909).

*A. ocellana (F.). Vi3.

$*$ A. propinquella $(\mathrm{Tr}$.). Ve1.

* Depressaria badiella (Hbn.). Vi3.

*D. depressana (F.). P13, Pr16.

*D. leucocephala Snell. Vi3.

D. pimpinellae Z. Kt1 (Krulikovsky 1909).

D. daucella (Den. \& Schiff.). Kt1, Vi3.

*D. sordidatella Tengstr. Pi4, Vi3.
*Ethmia quadrillella (Goeze). Us6.

*Elachista adscitella Stt. P14, So6.

*E. albifrontella (Hbn.). Ka14, Pi3, P12-3, Pr1, Pr16, Us4, Ve1.

*E. alpinella $\mathrm{Stt}$. Kh4, Kt8, P12.

*E. apicipunctella $\mathrm{Stt}$. Pr1, Ve1.

*E. diederichsiella E. Hering. Pr16, So5, Ve1.

*E. humilis Z. Pr16.

*E. maculicerusella (Bruand). So6.

*E. nobilella Z. Pi2, Pr1, Pr16.

*E. pullicomella Z. Ka1, Kh4, Pi3, Vi2.

*E. subalbidella Schläger. Ka10-11, Kh12, Kt2, $\mathrm{P} 13$, So6.

*E. tengstromi Kaila, Bengtsson, Šulcs \& Junnilainen. P17.

*E. baltica Hering. P12-3, Pr1, Pr16.

*E. eleochariella (Stt.). Kh4, Kh10.

*E. occidentalis Frey. Vi2.

*E. exactella (H.-S.). Pr1.

* Heinemannia laspeyrella (Hbn.). Ko3.

*Hypercallia citrinalis (Scop.). Kb4.

Stathmopodidae

*Stathmopoda pedella (L.). Kb4, Kt6.

Batrachedridae

Batrachedra praeangusta (Haw.). Kt5, Pr9, Pr16, Ve3.

Coleophoridae

Coleophora serratella (L.). Ka10, P12, Pr1, Pr16.

*C. milvipennis Z. Ko2.

*C. gryphipennella (Hbn.). Pr1.

*C. plumbella Kanerva. Pr16, Ve7.

C. lusciniaepennella (Tr.). Pr1 (Zelenova 1972).

$*$ C. potentillae Elisha. Ka14.

${ }^{*}$ C. violacea (Ström). So6.

C. binderella Kollar. Pr16.

C. sibiricella Falk. Pr1, Pr16. Older reports of $C$. laricella in the northern Russia refer to this species.

*C. cornutella (H.-S.). So6.

${ }^{*}$ C. albidella (Den. \& Schiff.). Ka1.

C. alcyonipennella (Koll.). Ka14, Kt1, Sh2, So56, Vi3, Vt6.

C. deauratella Lienig \& Z. Ka1, Ka7, Ka9-10, Ka12, Ka14, Kt1, Pi2, Pl2, Pr1, Pr16, Sh2, So5-6, Us4-5, Ve1, Ve8, Vi6.

${ }^{*}$ C. frischella (L.). Ka10.

*C. mayrella (Hbn.). Ka1, Ka8. 
*C. adjunctella (Hodgkinson). So6.

*C. alticolella Z. Ka1, Ka11, Ka13, Me2, So5, So7, P13, Ve1.

*C. glaucicolella Wood. Ka1, Ka11, Ve1, Vi2, Vi8.

C. directella Z. Kt1 (Krulikovsky 1909).

*C. striatipennella Nyl. So5-6, Ve8.

$*$ C. atriplicis (Meyr.). So5, So7.

*C. sternipennella (Zett.). Ve1.

$*$ C. virgaureae (Stt.). So6.

Momphidae

Mompha idaei (Z.). Kt1, Ko3, Pr16, So6, Us5, Vi7.

*M. conturbatella (Hbn.). Kh8, Pi4, P17.

*M. sturnipennella (Tr.). Ka10, Pr1, Pr16.

*M. locupletella (Den. \& Schiff.). P12.

*M. raschkiella (Z.). Ka6, Ka11, Kh4, Kh12, So6-7.

Scythrididae

* Scythris disparella (Tengstr.). Ka12.

$*$ S. inspersella (Hbn.). Ka11, P12.

*S. limbella (F.). Ka1.

S. noricella (Z.). Kt1, P17.

S. obscurella (Scop.). Ka1, Ka7, Ka10, Ka12,

Ka14, Ko2, Kt1, Us4-5, Ve1, Ve8.

*S. palustris (Z.). Vt5.

Cosmopterigidae

* Pancalia leuwenhoekella (L.). Pr16.

*Cosmopterix orichalcea Stt. Ka7, Ka10, Ka12, Ko3, Us4.

${ }^{*}$ C. sibirica Sinev. Ka14. A recently described species which has been recorded in Central Volga region (Sinev 2008) and found as far west as in Latvia and Estonia (Savenkov \& Šulcs 2010, Jürivete 2012).

Gelechiidae

Metzneria lappella(L.). Ka1, Kt1.

*M. metzneriella (Stt.). Ka1, Ka11, Ko2-3.

M. neuropterella (Z.). Kt1 (Krulikovsky 1909).

Isophrictis striatella (Den. \& Schiff.). Kt1 (Krulikovsky 1909).

*Argolamprotes micella (Den. \& Schiff.). Kb4, Vi3.

*Eulamprotes unicolorella (Dup.). Ka8, Ka12, Us4-5.
*Xystophora pulveratella (H.-S.). Ka7, Ka10, Ka14, Us4.

*Bryotropha senectella(Z.). Kb4, Kt5, Pr16, Vi5, Vt6.

*B. similis (Stt.). Ka1, Pi4, Pr16, Vi8.

*Gelechia sororculella (Hbn.). Ka1, Kt4, Ve1, Vi3.

Chionodes continuella (Z.). Ko2, Kt1.

Ch. distinctella (Z.). Kt1, So4, So6.

*Ch. fumatella (Douglas). Pr16.

*Ch. holosericella (H.-S.). P12.

*Ch. lugubrella (F.). P12, Pr16.

Ch. viduella (F.). P12, Pr16, Kt1.

Aroga velocella (Z.). Kt1, Vi3.

Filatima incomptella (H.-S.). P12, Pr16.

*Athrips tetrapunctella (Thnbg.). Ka10, Ka12.

* Gnorimoschema robustella (Stgr.). Kt4. This is one of the most interesting records of this study. The only specimen, a male, was collected running on bare sandy shore on the riverside. The species is previously known only from the steppe zone in Krasnoarmeysk, Saratov oblast of Russia and Uralsk (Lake Indersky) in Kazakhstan (Huemer \& Karsholt 2010).

*Scrobipalpa atriplicella (F. v. R.). Ka1, Ve1, Vi3.

* Klimeschiopsis kiningerella (Dup.). Pr16.

* Caryocolum blandella (Douglas). Pr16.

*C. cassella (Walk.). P110.

${ }^{*}$ C. petrophila (Preissecker). P110.

$*$ C. leucomelanella (Z.). Pi4. This is the northernmost record of this Euro-Siberian species, which is widely distributed in Central Europe, but does not occur in the Nordic countries (Huemer \& Karsholt 2010). The nearest records are from north-western region of Russia (Sinev 2008).

${ }^{*}$ C. vicinella (Douglas). Ka11.

Exoteleia dodecella (L.). Kt1 (Krulikovsky 1909).

Carpatolechia alburnella (Z.). Kt1 (Krulikovsky 1909).

${ }^{*}$ C. epomidella (Tengstr.). P13.

*C. notatella (Hbn.). Ka7, Pr16.

C. proximella (Hbn.). Ka6, P13, Pr1, Pr16, So6.

* Pseudotelphusa paripunctella (Thnbg.). P13, Pr1, Ve8.

Syncopacma cinctella (Cl.). Ka1, Ka12, Ka14, Kb4, Kt1, P13, Pr1, Pr16, So4, So6, Vi7, Vt4, Vt6. 
*S. karvoneni (Hackman). Ka1, Ka7, Ka10, Ka12, Ka14.

${ }^{*}$ S. sangiella (Stt.). Kh8, Kt5.

*Anacampsis blattariella (Hbn.). Kt4, P110, Pr1, Pr16.

A. populella (Cl.). Kb2, Kt1, Kt4-5, Kt7, On6, P14, Pr1, Pr16, Ve3, Vt3.

*A. temerella (Lienig \& Z.). Ka1, Kh10, Kt5, Pi6, P13, Pr16, Vt3.

* Prolita sexpunctella (F.). Pr1, Pr16, So1, Us3.

*Helcystogramma lineolella (Z.). P13, Pr16.

Acompsia cinerella (Cl.). Ka14, Ko2, Kt1, P13, Pr16, Ve8.

*A. subpunctella Svensson. Pr16, Vi2, Vi8.

* Dichomeris juniperella (L.). P12.

*Neofaculta infernella (H.-S.). P12-3, Pr1, Pr16, So4, So6-7.

*Hypatima rhomboidella (L.). Kh9, Pr16.

Pterophoridae

* Gillmeria pallidactyla (Haw.). Ka5, Kb4, Kh3, P17-8, Pr9, Pr12, Pr28, So8, Ve3, Vi3, Vi5, Vi8, Vt4, Vt6.

*G. tetradactyla (L.). Kh8, On3, Pi4, P17, Pr12, Ve5, Vi2, Vi8, Vt6.

Platyptilia calodactyla (Den. \& Schiff.). Kh6, Kt1, Kt5, Kt7, On6, Pi4, Pr10, Ve1, Vi7.

P. gonodactyla (Den. \& Schiff.). Ka11, Ka13-14, Kh12, Ko3, Kt1-2, Ny1, Pi4, Pr1, Pr28, Ve8, Vi5-6.

*P. tesseradactyla (L.). So0.

*Amblyptilia punctidactyla (Haw.). Ka13, Kt2, Ny1, Pi6, P18, Pr16, Sh2, So5-6, Us3-4, Us6.

*Stenoptilia bipunctidactyla (Scop.). Ka7, Vi3, Vi5.

*S. pterodactyla (L.). Kb2, Kb4, Kh6, Kh8, Kt45, Pi4, Pl10, Pr12, Vi5, Vi8, Vt3, Vt6.

*Geina didactyla (L.). Ka11.

Pterophorus pentadactyla (L.). Kt1 (Krulikovsky 1909).

*Hellinsia didactylites (Ström). Ka8, Ka11, Ka14, Kb4, Kh15, Ko2-3, Ny1, Pr28, So5-6, Us4, Ve4, Ve7, Vi6.

*H. osteodactylus (Z.). Kh15, Kt5, Pi2, Pr16, So4-6, Vi3, Vi5, Vi8.

*H. tephradactyla (Hbn.). P12, Ve8.

Emmelina monodactyla (L.). Kt1 (Krulikovsky 1909).
Schreckensteiniidae

* Schreckensteinia festaliella (Hbn.). Pr16.

Epermeniidae

* Phaulernis fulviguttella (Z.). Pr16.

* Epermenia chaerophyllella (Goeze). So5-6.

*E. illigerella (Hbn.). Pr16.

Choreutidae

*Anthophila fabriciana (L.). Ka5, Kh8, On3, Pi4, P17, P110, Pr1, Pr9, Pr16, Vi6.

*Prochoreutis ultimana (Krul.). Ka11, Vi2, Vi8.

*Tebenna bjerkandrella (Thnbg.). P17.

Choreutis diana (Hbn.). Ka4, Kb4, Kh6, Kt1, Kt7, Le2, On3, Pi5-6, P12, P18, Pr10, Sh2, So0, Ve2, Vi5, Vt3.

Tortricidae Acleris aspersana (Hbn.). Pr1, Pr6, Pr16.

*A. comariana (Lienig \& Z.). P13, Vi3, Vi6.

A. emargana (F.). Pl2, Pr1, Pr16, Vi3.

*A. effractana (Hbn.). Pl2, Pr1.

A. hastiana (L.). Le2, Pr16, Vi3.

*A. hyemana (Haw.). Pr16.

A. laterana (F.). Pr1, Pr16, Vi3.

A. lipsiana (Den. \& Schiff.). Pr1, Pr16, Vi3.

A. logiana (Cl.). Kt1, Pr1, Pr23, So1, Vi3.

A. maccana (Tr.). Pr16.

A. notana (Don.). Kt1, Pr1, Pr16, Pr23.

*A. rufana (Den. \& Schiff.). Vi3.

A. variegana (Den. \& Schiff.). Pr1, Pr16.

A. bergmanniana (L.). Kt1, P13, Pr1, Pr16.

Phtheochroa inopiana (Haw.). Ka1, Kt1, Pr16.

Agapeta hamana (L.). Kt1 (Krulikovsky 1909).

*Aethes cnicana (Westwood). Ka11.

*A. deutschiana (Zett.). Pr16.

A. hartmanniana $(\mathrm{Cl}$.). $\mathrm{Kt1}$, Us4, Ve7.

*A. rutilana $(\mathrm{Hbn}$.). P12.

*A. smeathmanniana (F.). Pr1, Pr16, So6, Ve8.

*A. triangulana (Tr.). Pl3, Pr16.

* Cochylidia implicitana (Wck.). Pr16.

${ }^{*}$ C. subroseana (Haw.). Pi2, P12-3, Pr9, Pr16, So5.

* Cochylis dubitana (Hbn.). P12, Pr16.

*C. nana (Haw.). P12, Pr1, So6-7.

*C. pallidana Z. Ka10, Ka14, Pr16.

Eulia ministrana (L.). Ka1, Kt1, P12-3, Pr1-2, Pr16, So1, So4-6, Vi6.

*Sparganothis rubicundana (H.-S.). Kh6, Pr16.

*Eana argentana (Cl.). Kb4, Kt4-5, On5-6, Pr1, 
Pr16, So6, Ve5, Vi3, Vt3, Vt5-6.

E. osseana (Scop.). Kh3, Kt1, On2, On7, Pi4, P12, P17, Pr11-12, So0, Vi3, Vi8.

*E. incanana (Steph.). Kt5.

*E. penziana (Thnbg.). P12, Pr16, Vi3.

Exapate congelatella (Cl.). P12, Pr1, Pr16.

Archips rosana (L.). Pr1, Pr12, Pr15-16, Pr24.

Argyrotaenia ljungiana (Thnbg.). Pr16.

Choristoneura albaniana (Walk.). Pr16.

Pandemis cerasana (Hbn.). Pr1, Pr16, So5.

*P. cinnamomeana (Tr.). Pr1.

P. heparana (Den. \& Schiff.). P13, Pr1, Pr16.

Syndemis musculana (Hbn.). Ka11, Kt1, P12-3,

Pr1-2, Pr16, So6.

Lozotaenia forsterana (F.). P12, Pr16, So1.

*Aphelia paleana (Hbn.). Ka1, Ka11-12, Kb2,

Kh8, Pi6, Pr1, Pr16, Ve3, Vi3, Vi5, Vi8, Vt4,

Vt6.

*A. unitana (Hbn.). Ve1, Ve4.

*A. viburnana (Den. \& Schiff.). On3, Vi3, Vi6-7.

*Clepsis rogana (Guenée). Ka1, Ka7-8, Ka10

12, Ka14, Ko2, Kh8, Us3-5, Ve4, Ve7, Vi5-6.

A boreomontane Euro-Siberian species (or a group of closely related species) found also in the Kola Peninsula (Kozlov \& Jalava 1994) but not elsewhere in the Nordic countries, except a few specimens from southeastern Finland (Ka: Virolahti). The latter record was published as Clepsis sp. nr. rogana (Kullberg et al. 2002, Hyönteistietokanta 2014).

C. senecionana (Hbn.). Kt1, P13, Pr1, Pr16, So4.

*Adoxophyes orana (F. v. R.). P19, Pr1.

*Epagoge grotiana (F.). So4, So6-7.

*Philedone gerningana (Den. \& Schiff.). Kh4, On3, Pr1, Vt5.

* Philedonides lunana (Thnbg.). Pr16.

*Endothenia ericetana (Humph. \& Westw.). Vi3, Vi5-6, Vt6.

*E. marginana (Haw.). Ka1.

*E. quadrimaculana (Haw.). Kt7, Pi4, Pr1, Vi3.

* Bactra furfurana (Haw.). Pi4, Pr11, Vi8, Vt4.

*B. lacteana Caradja. P13.

*B. lancealana (Hbn.). Kt4, Ve1, Ve2-3, Vi8. Apotomis betuletana (Haw.). Kt1, Pr1, Pr16.

A. capreana (Hbn.). Kt1, P17, Pr9-10, Pr16, Ve1.

A. infida (Heinrich). Pr1, Pr6, Pr16, Pr23.

*A. inundana (Den. \& Schiff.). Kt5.

*A. semifasciana (Haw.). Pr16.

A. sororculana (Zett.). Kt1, P12, Pr1, Pr16, Ve1.

A. turbidana Hbn. Pi2, P12, Pr1, Pr16.
Orthotaenia undulana (Den. \& Schiff.). Ka11,

Kh15, P12-3, Pr1, Pr16, Us4, Ve1.

* Pseudohermenias abietana (F.). P12-3.

*Hedya dimidiana (Cl.). Ka11, Ka14, Pr1.

H. nubiferana (Hw.). P12, Pr1, Pr16, Vi7.

*H. ochroleucana (Frölich). Kt5.

H. salicella (L.). Kt4, Pr1, Vi8.

H. atropunctana (Zett.). Ka1, P12-3, Pr1, So4, So6-7.

*Argyroploce arbutella (L.). So7.

*A. lediana (L.). P13, Us3, Vi5.

A. roseomaculana (H.-S.). P13, Pr16.

Olethreutes arcuella (Cl.). Ka13, Kh12, Kt1,

Ny1, Pi4, Pr16, Us4-5, Ve8, Vi6.

*Capricornia boisduvaliana (Dup.). Ka14.

Phiaris bipunctana (F.). P12-3, Pr16, Pr24, So1,

So4, So6-8, Us3.

*Ph. dissolutana (Stange). P12.

Ph. metallicana (Hbn.). Kt1, P12, Pr16.

Ph. micana (Den. \& Schiff.). Kt1, Pr16, Vt5.

*Ph. obsoletana (Zett.). So6, So8.

Ph. palustrana (Lienig \& Z.). Kt1 (Krulikovsky 1909).

*Ph. schulziana (F.). So1, So4, So6-8.

Ph. turfosana (H.-S.). Me1, P12, Pr16, So1.

*Ph. umbrosana (Freyer). Kb4, Pr16, So4, So67, Vi5.

Celypha cespitana (Hbn.). Kt1, Kt7, Vt5-6.

${ }^{*}$ C. rufana (Scop.). Kb2, Kb4, Kt4, P13, Pr16, Sh3, So6, Vi5, Vt3, Vt5.

*C. rurestrana (Dup.). Pr16.

C. striana (Den. \& Schiff.). Kb4, Kt4-5, Me1, P12, Pr1, Pr16, Us6, Ve1.

Loxoterma bipunctana (F.). P12-3, Pr16, Pr24, So1, Us3.

L. lacunana (Den. \& Schiff.). Ka4, Ka6-8, Ka10-11, Ka13-14, Kb2, Kb4, Kh6, Kh8, Kh10, Kh12, Kh15, Ko2-3, Kt1, Kt7, On3-4, On6, Pi4, Pi6, P12-3, P17, P110, Pr1, Pr10, Pr16, Pr24, Sh2, So4, So6, Us3-4, Ve1-5, Ve8, Vi2-3, Vi5, Vi7, Vt3-4.

L. rivulana (Scop.). Kb4, Kh3-4, Kt1, Kt5, Kt8, On3, On6, P13, P18, Pr12, Pr16, Ve2, Ve5, Vi5, Vi7-8, Vt3-6.

Pseudosciaphila branderiana (L.). Kt1 (Krulikovsky 1909).

*Eudemis porphyrana (Hbn.). Ka1, Kb4.

*Lobesia virulenta Bae \& Komai. Ka11, Pr16.

*Ancylis apicella (Den. \& Schiff.). Ka1, Ka8, Ka11, Ko3. 
A. badiana (Den. \& Schiff.). Ka8, Ka10-11, Ka14, Kb4, Kh6, Kh12, Ko2-3, Kt1, Me3, Ny1, Pi2-3, Pr1-2, Pr9, Pr16, So4, So6-7, Us3-4, Us6, Ve1, Ve4, Ve7, Vi6.

*A. comptana (Frölich). Ko2, P12, Pr1, Ve1.

*A. diminutana (Haw.). P13, Pr1, Pr16.

A. geminana (Don.). Pr1, Pr16.

A. laetana (F.). P12-3, Pr16, So4, So6, Ve1, Ve4. A. myrtillana (Tr.). P13, Pr1-2, Pr16, So4, So6.

*A. subarcuana (Douglas). Ka11, Pr16.

A. uncella (Den. \& Schiff.). P13, Pr16.

*A. unculana (Haw.). Ka11.

A. unguicella (L.). Ka7, Kt1, P12-3, Pr1-2, Pr16, So7, Ve7-8.

*Eucosmomorpha albersana (Hbn.). Ka1.

* Gypsonoma nitidulana (Lienig \& Z.). Pr16, $\mathrm{Ve} 2$.

G. sociana (Haw.). Pr1, Pr16.

Epinotia bilunana (Haw.). Pr1, Ve1.

E. brunnichiana (L.). P12, Pr16.

*E. caprana (F.). Pr1, Pr6, Pr16.

E. crenana (Hbn.). Pi5, P12, Pr1, Pr6, Pr16.

E. cruciana (L.). Pi4, P12-3, Pr1, Pr11, Pr16, Pr24, Vt3.

E. gimmerthaliana (Lienig \& Z.). P12-3, Pr16.

E. immundana (F. v. R.). P12-3, P17, Pr1, Pr16.

E. maculana (F.). P12, Pr6, Pr16.

E. nanana (Tr.). Pi6, P12-3, Pr10, Pr16, Pr24.

E. nisella (Cl.). Ka1, Ka6, Kt1, Kt5, P14, Pr1, Pr16, Vi3.

E. ramella (L.). Pr1, Pr6, Pr16.

*E. signatana (Douglas). Ve3.

E. solandriana (L.). P13, Pr1, Pr6, Pr16, Vi3.

E. subocellana (Don.). Kh2, Me3, Pi2, P13, Pr1, Pr16, So6-7.

E. tedella (Cl.). Kh12, Pi4, P12-3, Pr16, Pr24, Us3, Ve8.

E. tenerana (Den. \& Schiff.). Ka5-6, Kb2, Kt4, On4, Pi6, P12, Pr1, Pr9, Pr16, Ve3, Vi8.

E. tetraquetrana (Haw.). Ka7, Ka11, Kh2, Me3, Pi2, P12-3, Pr1-2, Pr16, So1, So5, Ve1.

*E. trigonella (L.). P12.

* Spilonota laricana (Hein.). Pr16.

Rhopobota naevana (Hbn.). Ka1, Kh4, Kh6, On3, Pi4, P14, Pr10, Pr16, Sh1, Ve5, Vi3.

Rh. ustomaculana (Curtis). Kh13, P12, Pr10, Pr16, Sh2, Vi2-3, Vi7.

Retinia resinella (L.). Pr16, So6.

Coccyx posticana (Zett.). Pr16.

C. turionella (L.). P13, Pr16.
*Eriopsela quadrana (Hbn.). P13, Pr16.

* Thiodia citrana (Hbn.). So6.

Notocelia cynosbatella (L.). Ka8, P13, Pr1, Pr16, So5, Us4.

N. incarnatana (Hbn.). Kt5, Pi4, Pr16.

*N. roborana (Den. \& Schiff.). Ka1, Kb2, Kt5, Pr1, Pr16.

N. tetragonana (Steph.). Pi6, Pr16.

N. uddmanniana (L.). Kt1 (Krulikovsky 1909).

*Epiblema cirsiana (Z.). Pi6, Pl3, Pr10, Pr16, Vi2, Vi7.

E. foenella (L.). Ka1, Kt1, Vi3.

*E. grandaevana (Lienig \& Z.). Kt4.

*E. scutulana (Den. \& Schiff.). Ka11.

E. similana (Den. \& Schiff.). Kt1 (Krulikovsky 1909).

*E. sticticana (F.). Ka8, Ka13-14, Kh12, Ko3, Ny1, P13, Pr1, Pr16, Sh2, Us3, Us6.

*Eucosma aspidiscana (Hbn.). P12-3, Pr16, So1, Ve7.

E. cana (Haw.). Ka1, Kb4, Ko2, Kt1, Kt5, Pr16, So5, Us4, Ve5.

*E. hohenwartiana (Den. \& Schiff.). Pr16, Vi3.

*E. obumbratana (Lienig \& Z.). So8, Vi5.

* Dichrorampha acuminatana (Lienig \& Z.). P17, Vi2, Vt3, Vt6.

$*$ D. aeratana (Pierce \& Metcalfe). Ve8.

*D. agilana (Tengstr.). Ka12, Kb2, Kh10, Kt4, Pi4, P17, Us5, Vi2, Vi5, Vi8.

*D. consortana (Steph.). Vt3.

*D. flavidorsana Knaggs. Kh3, Pr10, Vi8.

*D. vancouverana McDunn. Kb4, Kt8, On7, Pi4, Pr16, So6, Ve5, Vi5, Vi8.

*D. nigrobrunneana (Toll). Kb4, Ve4. This is the northernmost record of this rare Euro-Siberian species which does not occur in Fennoscandia. The nearest records are from Estonia (Karsholt et al. 2013) and from the central European region of Russia (Sinev 2008).

*D. obscuratana (Wolff). So7-8.

D. petiverella (L.). Ka5, Ka7, Ka10, Kb4, Kh3, Ko3, Kt1, Kt4, Kt6, P17, Sh2, Ve8, Vt6.

*D. plumbagana (Tr.). Ka8, Ka10, Ka12, Ka14, Pr16, So5, So8, Ve8, Vi5.

D. plumbana (Scop.). Ko2, Kt1, Pi2, Pr16, Sh23, Us5, Ve1.

*D. sequana (Hbn.). Ka14. This is the northernmost record of the species. The species has been recorded also in the southern taiga region of Russia south of Arkhangelsk oblast 
(Sinev 2008) and in southern Sweden (Gustafsson 2014), but not in the Baltic countries (Karsholt et al. 2013).

*D. uralensis (Danilevsky). Us3-5. The earlier published records from Russia are from more southern regions (Sinev 2008), but the species was collected also near Krasnyi Kamen in Polar Ural (J.K., pers. obs.). In Northern Europe, the only record is from Latvia (Karsholt et al. 2013).

* Grapholita compositella (F.). Ka10-12, Ka14, Kb4, Kh8, Ko2, Kt3, Me3, Pi2, Pi4, P123, Pr1, Pr9, Pr16, So4-6, So8, Pr16, Us4-5, Ve5, Vi8, Vt3.

*G. jungiella (L.). P12, Pr16.

*G. orobana (Tr.). Kh3, Pr16, So6.

* Pammene aurana (F.). Ka14, So4.

*P. clanculana (Tengstr.). Ve1.

*P. gallicana (Guenée). P12, P110.

P. germmana (Hbn.). Pr1, Pr16, Us6.

P. luedersiana (Sorh.). Pr16.

* Cydia cognatana (Barrett). Pr16.

*C. coniferana (Saxesen). P13, Pr16.

C. cornucopiae (Tengstr.). Pr16.

C. cosmophorana (Tr.). Pr16.

${ }^{*}$ C. illutana (H.-S.). Pr16, So1, So7.

C. indivisa (Danilevsky). Pr16.

*C. nigricana (F.). Ka7, Ka10, Ka14, Kh8, Ve1, Ve4-5, Vi8.

*C. pactolana (Z.). P13, Pr16.

*C. pomonella (L.). Ar0.

C. strobilella (L.). P12, Pr16.

C. zebeana (Ratz.). Ar0 (Danilevsky \& Kuznetzov 1968). This is the westernmost North European record of this Euro-Siberian species that occurs in Central Europe (Karsholt et al. 2013) and in Ural Mts. (Sinev 2008).

Lathronympha strigana (F.). Ka1, Ka14, Kb2, Kb4, Kh8, Kt1, On6, P110, Pr16, Vi3, Vi5, Vi8, Vt6.

Cossidae

Cossus cossus (L.). Kh15, Kt1, P13, Pr1, Vt2.

Sesiidae

Paranthrene tabaniformis (Rott.). Pr1 (Zelenova 1972).

*Synanthedon formicaeformis (Esp.). Pr16, Pr28, Us4.
S. scoliaeformis (Borkh.). Pr1 (Zelenova 1972).

S. spheciformis (Den. \& Schiff.). Pr16 (Zelenova 1972).

S. tipuliformis (Cl.). Pr1, Pr16 (Zelenova 1972).

Zygaenidae

Zygaena osterodensis Reiss. Ka15, Pi4.

*Z. viciae (Den. \& Schiff.). Kb4.

$*$ Z. filipendulae (L.). Ka0.

*Z. lonicerae (Scheven). Kb4, Pr11.

Thyrididae

* Thyris fenestrella (Scop.). Pr16. This is the northernmost record of the species.

Papilionidae

Parnassius mnemosyne (L.). Ko2, Le3, Me5, Me6, Pi4, Pi8, Pr35, Us1, Us3-5, Vi9.

Papilio machaon L. Ka14, Kh15-16, Kt1, Me5, On0, Pi3-4, Pi7, Pr1, Pr5, Pr9, Pr16-17, Pr19, Pr22, Pr29-30, Pr32, Sh4, So0, Vi4.

Hesperiidae

* Pyrgus alveus Hbn. Ve8.

*P. centaureae (Rambur). P13, So0.

P. malvae (L.). Ka10, Kt1, Us3, Us4.

P. serratulae (Rambur). Kt1 (Krulikovsky 1909).

The nearest records are from Vologda and Ukhta (Lvovsky \& Morgun 2007). The species is present in Baltic countries, but does not occur in Fennoscandia (the record by Karsholt et al. [2013] from Sweden is erroneous).

Carterocephalus palaemon (Pallas). Pi4, P13, Pr1, Pr5, Pr13, Pr16-18, Pr32.

C. silvicola (Meigen). Pr0, Us4-5.

Thymelicus lineola (Ochs.). Ka5, Kb2, Kb4, Kh8, Kh10, Kt1, Kt3-5, Kt8, On6-7, P14, P18, Pr1, Pr12, Sh2, Sh4, Ve2-3, Ve5, Vi2-3, Vi5, Vi7$8, \mathrm{Vt3}, \mathrm{Vt5}-6$.

Ochlodes sylvanus (Esp.). Ka7-8, Ka10-12, Ko2, Kt1, Pr0, Ve4.

Hesperia comma (L.). Kt1 (Krulikovsky 1909).

Pieridae

Leptidea morsei (Fenton). Ka11, Ka14, Kh8, Pi4, Pr1, Pr9, Pr16, Pr17, Us2-3, Us5-6, Ve6, Ve8, Vi10. This Euro-Siberian species has not been recorded west of Arkhangelsk oblast in 
the northern Europe (Sinev 2008, Karsholt et al. 2013).

L. sinapis (L.). Ka13, Kh12, Kh15, Me2, On8, Pi4, Pi9, Pr1, Pr9, Pr13, Pr16-18, Sh4, So1, So6, Us3-6, Ve8, Vi10.

Anthocharis cardamines (L.). Ka14, Kh15-16, Kt1, Pi4, Pr1, Pr12-13, Pr26, So0, So6, Us5.

Aporia crataegi (L.). Ka7, Ka10-14, Kb3, Kh16,

Ko2, Kt1, On0, Pi4, P12, P16, Pr1, Pr16, Sh4,

So0, So6, Us3-6, Ve5, Vt2.

Pieris brassicae (L.). Le3, Pi4, P10, Pr16, Vi4.

P. napi (L.). Ka1, Ka4, Ka6, Ka15, Kb4, Kh8, Kh15, Ko2, Kt1, On6, Pi4, Pr1, Pr9, Pr13-14, Pr16, Sh2, Sh4, So0, So8, Us4, Us6, Ve3, Vg1.

P. rapae (L.). Ka15, Kh7, Kh15, Kt1, On7, Pi4, P16, Pr1, Pr3, Pr5, Pr12, Pr14, Pr16-17, Pr21, So0, Us4, Ve1, Vg1, Vi3.

Pontia daplidice (L.). Ka4, Ka6, Kh15, Kt1, On7, P18, P110, Sh2, So0, Vi3.

Colias hyale (L.). Kt1, On6, Pi4.

C. palaeno (L.). Ka2, Kh7, Kh15, Kt1, Me4, Pi3, Pi4, Pr4, Pr21, So0, So4, So6-8, Vt2.

Gonepteryx rhamni (L.). Ka2, Ka4-6, Ka15, Kb2, Kh8, Kh10, Kh15, Kt1, Kt3-5, Kt7, On3-4, On6-7, Pi4, Pi6, P14, P16-8, P110, Pr10-12, Sh2, Sh4, So0, Us4-6, Ve2-3, Ve5, Vg1, Vi2-3, Vt2.

Lycaenidae

Thecla betulae (L.). Kt1 (Krulikovsky 1909).

Satyrium pruni (L.). Ktl (Krulikovsky 1909).

Callophrys rubi (L.). Le3, Pr1, Pr12-13, Pr16, Pr27-28, So1, So6, Us3, Vt2.

Lycaena helle (Den. \& Schiff.). Ka14, Kh16, Le3, Pi4, P13, Pr5, Pr13, So1, Vt2.

L. hippothoe (L.). Kh16, Kt1, Le3, Pr26, So0, So4, So6, Ve5.

L. phlaeas (L.). Kt1, Sh4, Vg1.

L. virgaureae (L.). Kb4, Kh15-16, Kt1, Le3, On4, On6, Pi4, P17-8, Pr1, Pr9, Pr11-12, Sh2, Sh4, So1, Vi3, Vt3, Vt6.

Cupido minimus (Fuessly). Kh15-16, Pi4, Pr27.

C. alcetas (Hoffmannsegg). Pi4, Vi3. These are the northernmost records of this boreomontane Euro-Siberian species, which has only once been found in Fennoscandia (SE Finland: Kolev \& Kullberg 2000).

$* C$. argiades (Pallas). Sh4, Us4.
Celastrina argiolus (L.). Kh16, Me3, Pi3-4, Pr1, Pr16, Pr19, Pr26-27, Pr32, So1.

Glaucopsyche alexis (Poda). Kh16, Pr9, Pr12, So0, Us3.

Plebeius argus (L.). Kb4, Kh4, Kh16, Kt1, Le3, On3-4, Pr3, Pr27, So0, Ve2, Ve4, Vt5.

P. idas (L.). Kb4, Kh15, Kt4, Le3, On3, Pi6, Pr10, Pr12, Pr27, So1.

P. optilete (Knoch). Kb2, Kh10, Kh15, Le3, Pi4, Pr3, Pr11, Pr26, So4, So6-8, Ve4, Vi2, Vt5.

Aricia agestis (Den. \& Schiff.). Kt1 (Krulikovsky 1909).

A. $\operatorname{artaxerxes}(\mathrm{F}.) . \mathrm{Kh} 8, \mathrm{Kh} 15, \mathrm{On} 6, \mathrm{Pi} 4, \mathrm{Pr} 16$, Sol.

A. eumedon (Esp.). Pi4, So0, Ve4.

A. nicias (Meig.). Pi4 (Tikhomirov \& Bolotov 2000).

Polyommatus amandus (Schn.). Ka14, Kb4, Kh5-8, Kh15, Ko2, Pi4, Pi6, Pr1, Pr3, Pr5, Pr12, Pr17, Pr21, Sh2, Us4, Ve1, Ve5, Vi2, Vi8.

P. icarus (Rott.). Ka5, Kb2, Kb4, Kh3, Kh5-10, Kh16, Ko2, Kt1, Kt4, Le3, On3-4, On6, Pi4, Pi6, P12, P14, P16, P18, P110, Pr1, Pr9-10, Pr16, Pr21, Sh2, Sh4, So1, So4, So6, Us3-4, Ve1, Vi3, Vt3, Vt5-6.

Cyaniris semiargus (Rott.). Ka6-7, Ka11, Ka14, Kb2, Kb4, Kh10, Kh16, Kt1, Kt4, Le3, On4, Pi4, Pr1, Pr12, Pr17, Pr21, Pr26, So4, So6, So8, Us5, Ve1, Ve5, Vi2-3, Vi8, Vt3, Vt6.

Nymphalidae

Limenitis populi (L.). Kh15, On0, Pi4, P14, P16, Pr3, Pr21, Vi4.

*Neptis rivularis (Scop.). Kb4, Us3-4, Vg1. The species is locally abundant in habitats where its foodplant Spiraea is present. The species has been reported from north-western and Kaliningrad regions of Russia (Sinev 2008), but it is absent from the Baltic and the Nordic countries (Karsholt et al. 2013).

Neptis sappho (Pallas). Vt2 (Filippov 2009). The distribution of the species is very similar to that of the previous species, although $N$. sappho is absent from the north-western region of Russia (Sinev 2008). The species is much scarcer than the similar $N$. rivularis, probably due to the scarcity of its food plants Lathyrus vernus and L. niger. 
Nymphalis antiopa (L.). Ka2-5, Kh15, Kt1, Pi4, P16, Pr8, Us4, Vi3-4, Vt2.

Nymphalis xanthomelas (Esp.). Kt1, Sh4.

Aglais urticae (L.). Ka1, Ka3, Ka7, Kb2, Kh3, Kh5, Kh15, Kt1, On0, Pi3-4, P14, P16, Pr1, Pr12, Pr21, Sh3-4, So0, So6, Vi1, Vg1, Vi3, Vt2, Vt6.

A. io (L.). Ka3, Ka5, Kh15-16, On6, Pi3-4, P10, Pr28.

Polygonia c-album (L.). Ka4, Ka15, Kb4, Kh5, Kh16, Kt1, On6, Pi4, P14, P16, P18, Pr8, Sh4, So0, Ve9, Vg1, Vi3.

Vanessa atalanta (L.). Kh15-16, On0, Pi3-4, P10, Pr1, Pr5, Pr17, So0, So4.

V. cardui (L.). Ka14, Kh15, Kt1, Pi4, Pr1, Pr3, Pr5, Pr16-17, Pr19, Pr21, Pr32, So0, So4, So8.

Araschnia levana (L.). Ka13, Kh7, Kh15, On3, On6, Pr20, Pr26, Sh4, So0, So4, Vi3.

Euphydryas maturna (L.). Kh15-16, Ko2, Pi3-4, Pr1, Pr9, Pr21, Us3-5, Vt2.

Melitaea diamina (Lang). Ka12, Ka14, Ko2, Kt1, Us3, Ve8.

*M. phoebe (Den. \& Schiff.). Ve8. This is the northernmost record of the species. It has been reported from all Baltic countries, but not from north-western region of Russia (Sinev 2008, Karsholt et al. 2013).

M. athalia (Rott.). Kh15, Ko2, Kt1, Pi4, Ve8.

*Boloria dia (L.). Sh4. This is the northernmost record of the species. It was reported as expansive in the Baltic countries (Jürivete \& Õunap 2008).

B. eunomia (Esp.). Ka14, Pr1, Pr18, Sh1.

B. euphrosyne (L.). Ka7, Kh15, Ko3, Kt1, Pi4, Pr1, Pr13, So0, So6-7, Us3-4, Ve4.

*B. freija (Thnbg.). Ve4.

B. selene (Den. \& Schiff.). Ka11, Kb4, Kh7, Kh15, Ko3, Kt1, Le3, Pi4, Pr0, So0, So4, Us3-4, Ve5, Vi2.

B. titania (Esp.). Ka15, Kt1, Le3, Pi4, Pr0, Ve2.

B. aquilonaris (Stich.). Kh15, Le3, Pi4, Pr5, Pr13, So0.

Brenthis ino (Rott.). Ka5, Kb4, Kh7-8, Kh10, Kh15-16, Kt1, Le3, On4, On6, Pi4, Pi6, P14, P16-7, Pr3, Pr5, Pr9-10, Pr14, Pr21, Sh2, Ve2-5, Vi2-3, Vi8, Vt3-4, Vt6.

Issoria lathonia (L.). Kt1, Pr0.

Argynnis adippe (Den. \& Schiff.). Ka2, Kh16, P14, Pr0, Sh4, Vi3, Vt6.
A. aglaja (L.). Ka6, Kh16-17, Kt1, Kt4-5, Le3, On6, Pi3, P14, P16, Pr17, Pr21, Pr32, Ve5, Vi2-3, Vt3.

A. niobe (L.). Pr0.

A. paphia (L.). Ka2, $\mathrm{Ka} 4, \mathrm{Ka} 15, \mathrm{~Kb} 2, \mathrm{~Kb} 4, \mathrm{Kh} 7$, Kh16, Kt1, Kt4, On6, Pi4, P14, Pr16, Pr32, $\mathrm{Sh} 4, \mathrm{Vi2}, \mathrm{Vt} 3$.

Pararge aegeria (L.). Kt1 (Tatarinov \& Dolgin 1999), So0 (Bolotov et al. 2013a).

Lasiommata maera (L.). Kh16, Kt1, Pi4, Vi7.

L. petropolitana (F.). Pi4, Pr1, Pr13, So0.

Coenonympha glycerion (Borkh.). Ka7, Ka14, Kb4, Kh16, Ko2, Kt1, P19, Pr11, Ve8.

C. pamphilus (L.). Kt1, Pr11.

C. tullia (Müll.). Le3, Pi4, Pr1.

Aphantopus hyperantus (L.). Ka1, Kb2, Kb4, Kh7-8, Kh16, Kt1, Kt5, On3, P14, P18, Sh2, Sh4, Ve1, Ve5, Vg1, Vi3, Vi8, Vt3, Vt6.

Maniola jurtina (L.). Kb2, Kb4, Kh15-16, Kt1, $\mathrm{Kt} 4, \mathrm{P} 16, \mathrm{Sh} 2, \mathrm{Vt} 3$.

*Hyponephele lycaon (Rott.). Kt5.

Erebia disa (Thnbg.). Me1 (Poppius 1906).

E. embla (Thnbg.). Kh5, Kt1, Pr0, So0.

E. euryale (Esp.). Ka15, Kh15, Kt1, Le3, Pi4, $\operatorname{Pr} 17$, Vi2-3. This is a boreomontane species, which does not occur in western Fennoscandia. From the Russian Karelia eastwards it occurs as ssp. euryaloides Tengström. There is only one record inside the current borders of Finland (Sb: Lieksa, Välimäki et al. 2008).

E. ligea (L.). Ka6, Kb4, Kh5-6, Kh8, Kh15-16, Le3, On6, Pi3-4, P14-5, P17, Pr3, Pr10-11, Pr16-17, Pr21, So0, Ve2, Vi2-3, Vi7, Vt3. Oeneis bore (Schn.). Pr0 (Tuzov et al. 1997).

O. jutta (Hbn.). Pi4, Pr16-18, So0.

Pyralidae

Aphomia sociella (L.). Ka1, Kt1.

Pyralis farinalis (L.). Kt1, Pr16.

Aglossa pinguinalis (L.). Kt1, Ve1.

Ortholepis betulae (Goeze). Ka1, Kt1, P13, Pr1, Pr16.

* Pyla fusca (Haw.). Ka1, Ka6, So6, Ve1.

* Catastia marginea (Den. \& Schiff.). Ka7, Ka14.

* Sciota fumella (Ev.). Ka1, Ka6, Ka11, Ve1.

*S. rhenella (Zinck.). Ve1.

Oncocera semirubella (Scop.). Ka1, Kt1, Kt4, On4, Vi3, Vt6.

*Dioryctria abietella (Den. \& Schiff.). Ka1.

*D. schuetzeella Fuchs. Vi3. 
Hypochalcia ahenella (Den. \& Schiff.). Ka12, $\mathrm{Kb} 4, \mathrm{Kt} 1, \mathrm{Kt} 4$.

*Episcythrastis tetricella (Den. \& Schiff.). Ka1.

*Phycitodes binaevella (Hbn.). Ka1, Ve1, Vi3.

*Ph. maritima (Tengstr.). Pr9.

* Plodia interpunctella (Hbn.). Pr1 (B. Yu. Filippov, pers. comm.).

* Ephestia elutella (Hbn.). Pr1, Pr16.

Scoparia ambigualis (Tr.). Kt1 (Krulikovsky 1909).

*S. ancipitella (La Harpe). Ka1, Ka6, Kh6, Kt4,

Kt7-8, On6, P17, P110, Sh2, Ve2-3, Vi3, Vi5,

Vi7-8, Vt3, Vt5.

*Eudonia alpina (Curt.). So6.

E. lacustrata (Panz.). Ka1, Ka4, Kt1.

*E. murana (Curt.). Ka1.

*E. pallida (Curt.). Ka1.

Gesneria centuriella (Den. \& Schiff.). Kt1

(Krulikovsky 1909).

* Donacaula mucronella (Den. \& Schiff.). Ka1.

Elophila nymphaeata (L.). Kt1, Vi8, Vt4.

* Parapoynx stratiotata (L.). Vt4.

Nymphula nitidulata (Hufn.). Ka1, Ka4-6, Kt1, Kt4, On4, Pi4, P18, Pr3, Pr10, Pr16, Vt6.

*Evergestis extimalis (Scop.). P13.

*E. forficalis (L.). Ka1.

*E. pallidata (Hufn.). Kb2, Kh3, On6-7, Pr3, Vi3, Vi5, Vt3-4.

Udea decrepitalis (H.-S.). Kt1, P12-3, Pr16, So4, So6-7.

*U. hamalis (Thnbg.). P12, Pr1, Pr24, So5-6.

*U. inquinatalis (Lienig \& Z.). So4, So7.

*U. lutealis (Hbn.). Ka1, Ka4-5, Kb2, Kb4, Kh3, Kh5, Me2, On3-4, On6-7, Pi4, Pi6, P14-5, P17, P18, P110, Pr1, Pr3, Pr9, Pr12, Pr16-17, Sh2, Ve1-2, Ve5, Vi2-3, Vi5, Vi8, Vt4, Vt6.

U. prunalis (Den. \& Schiff.). Kb4, Kt1, On6, So1, Ve5, Vi3.

Opsibotys fuscalis (Den. \& Schiff.). Ka8-11, Ka13, Kb4, Kh6, Kh15, Ko3, Kt1-3, Me3, Pr16, Sh2, So5-6, Ve4-5, Ve8, Vi6.

Loxostege sticticalis (L.). Kt1 (Krulikovsky 1909).

Pyrausta despicata (Scop.). Kt1 (Krulikovsky 1909).

P. porphyralis (Den. \& Schiff.). Pr16.

P. purpuralis (L.). Ka1, Ka5, Kb2, Kt1, Kt4, Kt8, Pi4, P13, P17, P110, Pr16, Sh2, So5, Us4, Ve78, Vt6.

*Nascia cilialis (Hbn.). Ka11, Pr1.
Sitochroa verticalis (L.). Ka1, Ka9, Kb4, Ko2, Kt1, Pr1, Ve4-5, Vi5.

* Phlyctaenia coronata (Hufn.). Pr16, So6, Vi2.

*Ph. perlucidalis (Hbn.). Ka8, Ko3, Ve4.

*Psammotis pulveralis (Hbn.). Kh8, Kt4, On7, Pr16, Vi8, Vt3-4, Vt6.

*Ostrinia nubilalis (Hbn.). Ka1, Ka12, Ka14, Ko2, Pr17.

* Anania funebris (Ström). Pr2, Pr16, So4-7.

*A. terrealis (Tr.). So5, So6.

A. hortulata (L.). Ka1, Ka13, Kt1, Pr1, Pr5, Pr9, Pr17, Sh3, So6.

* Paratalanta pandalis Hbn. Ka11, Pr16, Us6, Ve7.

*Pleuroptya ruralis (Scop.). Ka1, Ka5-6, Kb2, On6, P110, Ve5, Vi3.

*Diasemia reticularis (L.). Ka14.

Nomophila noctuella (Den. \& Schiff.). Kt1 (Krulikovsky 1909).

Crambidae

* Calamotropha paludella (Hbn.). Ve5.

Chrysoteuchia culmella (L.). Ka1, Ka7, Ka9-12, Ko2, Kt1, Ny1, Pr1, Pr9, Pr16, So4, So8, Us3-5, Ve1, Ve4, Ve7-8, Vi6.

*Crambus alienellus (Germar \& Kaulfuss). P12, Pr16, So4, So6-7, Us3, Ve2, Vt5.

*C. ericella (Hbn.). Ka1, So8, Ve4.

${ }^{*}$ C. hamella (Thnbg.). Ka1, Kh4.

${ }^{*}$ C. lathoniellus (Zinck.). Ka1, Ka7-8, Ka10, Ka13, Kh15, Ko2-3, P13, Pr1, Pr9, Pr16, Sh2, So4-6, Us4, Us6, Ve1-2, Ve4, Ve7, Vi3, Vt3.

C. pascuella (L.). Kt1 (Krulikovsky 1909).

C. perlella (Scop.). Kb2, Kb4, Kh10, Ko2, Kt1, Kt4-5, Pi4, Pr10-11, So1, So5-7, Ve2, Ve5, Ve8, Vi3, Vi5, Vi8, Vt3-4, Vt6.

C. pratella (L.). Kt1, On2.

*Agriphila inquinatella (Den. \& Schiff.). Kb4, $\mathrm{Kt8}$.

*A. selasella (Hbn.). Ka1, Ka5-6, On3-4, On7, P14, P17-8, P110, Ve2, Vt3.

*A. straminella (Den. \& Schiff.). Ka6, Kb2, Kb4, Kh3, Kh6, Kh8, Kh10, Kt4-5, Kt7, On3-4, On6, P17-8, P110, Pr1, Pr12, Sh2, So5, Ve12, Ve5, Vi2-3, Vi5, Vi7-8, Vt4-6.

A. tristella (Den. \& Schiff.). Ka1, Ka6, Kb2, Kb4, Kt1, On4, P14, P17-8, Sh2, Vi3, Vi5, Vt3-4.

* Pediasia truncatella (Zett.). P13, Pr16.

*Catoptria maculalis (Zett.). So7.

${ }^{*}$ C. margaritella (Den. \& Schiff.). Ka4, Kh6, 
Kt7-8, On3, Pi4, P12, Ve2, Vi2, Vt3.

*C. permutatella (H.-S.). Ka1.

*C. pinella (L.). Kb4.

Drepanidae

Falcaria lacertinaria (L.). Pi4, P13, Pr1, Pr16, So6, Vi6.

Drepana falcataria (L.). Kt1, Pi2, Pi4, P13, Pr1, Pr16, So0.

Thyatira batis (L.). Pr16, Sh4, Ve9.

*Habrosyne pyritoides (Hufn.). Sh4, Ve9.

*Tethea ocularis (L.). Sh4.

T. or (Den. \& Schiff.). Pr1, Pr16, Sh4.

Ochropacha duplaris (L.). Pi4, P13, Pr16.

Lasiocampidae

Trichiura crataegi (L.). Ka15, Kt1, P12, Pr16.

Poecilocampa populi (L.). Pi4, Vi3.

Lasiocampa quercus (L.). Pr1, Pr11-12.

Macrothylacia rubi (L.). Kt1 (Krulikovsky 1909), Pr1, Pr 13 (Bolotov et al. 1998).

Euthrix potatoria (L.). Ka15, Kt1.

* Gastropacha quercifolia (L.). Ar0.

Phyllodesma japonica (Leech). Pr16 (Zelenova

1972). This is a rare Euro-Siberian species occurring eastwards from the Baltic countries, in Karelian Isthmus, but not in Russian Karelia or Nordic countries (Kaisila 1962, Sinev 2008, Karsholt et al. 2013).

Endromididae

Endromis versicolora (L.). Kh15, Pr13.

Saturniidae

* Aglia tau (L.). P10.

Saturnia pavonia (L.). Pr16 (Zelenova 1972), Pr1, Pr17, Pr19, Pr21, Pr32 (Bolotov et al. 1998).

Sphingidae

Acherontia atropos (L.). Ar0, Pi0.

Sphinx pinastri (L.). Ar0.

* Smerinthus caecus Ménétriés. Ar0. This is the northernmost record of this Euro-Siberian species which is distributed from north-western Russia eastwards (Sinev 2008) but has not found in the Baltic countries or Fennoscandia.

Smerinthus ocellatus (L.). Pr1 (Bolotov et al. 1998).

Laothoe populi (L.). P12, Pr1, Pr3, Pr9, Pr16.

Hyles gallii (Rott.). Kt1, Pi3, Pi4, Pi7, Pr1, Pr5,
Pr16, Pr19, Pr33-34, So3.

Deilephila elpenor (L.). Kt1, Pi3, Pi4, Pr5, Pr17, Sh4, Ve9.

D. porcellus (L.). Ve1, Sh4.

Macroglossum stellatarum (L.). Pr1, Pr12.

Hemaris fuciformis (L.). Kt1, Pi4, Pr1, Pr13, Pr16, Pr32, Us6.

Geometridae

Archiearis parthenias (L.). Kh11, Kt1, Pr16.

*Abraxas sylvata (Scop.). Ka7-8, Ka11, Kb4, Sh4, Us2, Ve8.

Lomaspilis marginata (L.). Ka6-8, Ka10-14, Kh2, Ko2-3, Kt1, Ny1, On1, Pi4, P12-3, Pr1, Pr9, Pr16, Pr24, Pr28, So1, So4, So6, Us2-4, Us6, Ve1, Ve4, Ve7-8, Vi6.

*L. opis Butler. Us2.

* Lomographa bimaculata (F.). Ka13, Us4, Ve1.

*L. temerata (Den. \& Schiff.). Pr1, Sh4.

Cabera exanthemata (Scop.). Ka8, Ka11, Ka13, Kb2, Kh2, Kh12, Kt5, Kt7, Me3, Ny1, On3, Pi4, P12-3, P14, P17, Pr1, Pr9, Pr16, Sh2, So1, So4, So6-8, Us2-4, Us6, Ve1, Vi3, Vi5-6.

${ }^{*}$ C. leptographa Wehrli. Ka13. This is the northernmost record of this rare Euro-Siberian species. It has been previously recorded in the Baltic countries and in the north-western and north-central regions of Russia (Sinev 2008). In 2012, a single specimen was found in Finland (Ka: Virolahti; Hyönteistietokanta 2014).

C. pusaria (L.). Ka1, Ka7-8, Ka13-14, Ko2, Kt1-2, Pi2, Pi4, P12-3, Pr1, Pr9, Pr16, Pr24, So1, So4, So6-7, Us4, Us6, Ve1, Ve7.

*Ennomos autumnaria (Werneburg). Kh15.

Selenia dentaria (F.). Kt1, P12, Pr2, Pr16, Pr23, Sol.

*S. lunularia (Hbn.). Pr2.

S. tetralunaria (Hufn.). P12, Pr1, Pr16.

*Crocallis elinguaria (L.). Ka15, Sh4, Vi3.

Opisthograptis luteolata (L.). Kt1, On0, Pr1, So1, So6, Us3.

Plagodis pulveraria (L.). Kh15, Pi4, P12-3, Pr12, Pr16, So1, So4-7, Us3, Ve6.

*Cepphis advenaria (Hbn.). Ka7-8, Ka13, Ko2, Us2, Us6, Ve6.

Pseudopanthera macularia (L.). Ko2, Kt1.

Epione repandaria (Hufn.). Ka1, Ka6, Kh16, Pi4, P12, P17, Pr16.

E. vespertaria (L.). Ka15, Kb4, P12, Pr16. 
*Epirranthis diversata (Den. \& Schiff.). Kh11, Pr16.

*Hylaea fasciaria (L.). Ka15.

Macaria alternata (Den. \& Schiff.). Ka1, Ka4, Ka8, Ka15, Kt1, Pi4, P110, Pr1, Pr4, Pr16, Sh4, Vi3.

M. brunneata (Thnbg.). Ka3, Kh6, Kt1, On3, Pi4, Pi6, P12, Pr1, Pr15-16, Pr24, Sh2, So1, Ve2, Vi7, Vt5.

M. carbonaria $(\mathrm{Cl}$.). Me1, $\operatorname{Pr} 2$.

M. liturata (Cl.). Pi4, Pr12, Pr16.

M. loricaria (Ev.). Kb4, Kt1, Pi4, P12, Pr1, So0.

M. notata (L.). Ka1, Kh15, Ko2-3, Kt1, Pi4, Pr16, So6-7, Us3, Us6, Vi3.

M. signaria (Hbn.). Pr16, So6.

M. wauaria (L.). Ka1, Ka12, Ka15, Kb4, Kt5, Pi4, P12, Pr1, Pr16, Sh4, Vi3.

Chiasmia clathrata(L.). Ka7-8, Ka10-12, Ka14, Kb2, Kh2, Kh15-16, Ko2-3, Kt1-3, Pi2, Pi4, Pr1, Pr16-17, Pr25, Sh4, So1, So4, So6, Us26, Ve1, Ve4-6, Ve8, Vi6.

Hypoxystis pluviaria (F.). Kt1 (Krulikovsky 1909).

Siona lineata (Scop.). Ka7, Ka9-12, Ka14, Ko3, Kt1, Sh4, Us3-5, Ve1, Ve4, Ve6, Ve8.

*Deileptenia ribeata (Cl.). Ve9.

*Elophos vittaria (Thnbg.). So0.

Ematurga atomaria (L.). Ka8-11, Ka13, Kh15, Ko2-3, Kt1, Kt3, Me3, Pi4, P12-3, Pr1-2, Pr9, Pr16, Sh4, So1, So4, So6-7, Us2-5, Ve6-8.

Angerona prunaria (L.). Ka8, Ka10-12, Ka14, Kh16, Ko2-3, Kt1, Us5.

Arichanna melanaria (L.). Ka15, Kh6, On3, Pl2, Pr1.

*Alcis jubata (Thnbg.). Pi4.

A. repandata (L.). Ka15, Kh8, Kt1, P12, Ve9.

*Hypomecis roboraria (Den. \& Schiff.). Sh4, Ve9.

* Paradarisa consonaria (Hbn.). Us2, Ve6.

Ectropis crepuscularia (Den. \& Schiff.). Kh15,

P12, Pr1, Ve6.

*Biston betularia (L.). Ve1.

Lycia hirtaria (Cl.). Pr16 (Zelenova 1972).

L. pomonaria (Hbn.). P12, Pr16 (Zelenova 1972).

Geometra papilionaria (L.). Ka15, Kh6, Kh11,

Kt1, Kt5, Pi4, P12, Pr10, Pr16-17, Pr20-21,

Pr31, Ve3, Vg1, Vi3, Vt4.

*Thetidia smaragdaria (F.). Ve8.

Jodis lactearia (L.). P12, So1, Us2.
J. putata(L.). Kh9, Ko3, Kt1, Me3, Pi2, Pi4, P123, Pr11, Pr16, Pr25, So1-2, So4-6, Us2, Ve67, Vi6.

*Chlorissa viridata (L.). Us2, Us6, Ve6.

Idaea aversata (L.). Kt1 (Krulikovsky 1909), Pi4 (Antonova \& Tikhomirov 2002).

*I. biselata (Hufn.). Ka15, Kb2, Kb4, Kt7, Sh4, Vi3, Vi7, Vi8.

I. pallidata (Den. \& Schiff.). Ka8, Ka11, Ka14, Ko2, Kt1, P13, Pr1, Pr9, Pr16, Us3-4, Ve6-8, $\mathrm{Vt5}$.

*I. serpentata (Hufn.). Kb4, Kt4, Kt5.

Scopula floslactata (Haw.). Ka14, Kh15, Ko2, Kt1, Pr16, So1, Us2-3, Ve4, Ve6-7.

S. frigidaria (Möschl.). Pi4 (Antonova \& Tikhomirov 2002).

S. immorata (L.). Ka7-10, Ka12, Ka14, Ko2, Kt1, Sh4, Us3-5, Ve1, Ve4, Ve8.

*S. immutata (L.). Kb2, P13, So1, Us2.

S. rubiginata (Hufn.). Ka6, On4, Pi4, P12, Pr1, Pr16, Ve8.

S. ternata Schrank. Kh12, Pi4, P12-3, Pr1, Pr16, So1, So4, So6-7.

Cyclophora albipunctata (Hufn.). Pi4, P12, Pr1, Pr23.

C. pendularia $(\mathrm{Cl}$.). Kt1, Pr1.

*Timandra comae Schmidt. Ve6. The species is probably a newcomer in Arkhangelsk oblast. It has recently been expanding northwards in Finland (Hyönteistietokanta 2014).

T. griseata W. Petersen. Ka11, Kh16, Kt1, Vi3.

Scotopteryx chenopodiata (L.). Ka4, Ka6, Ka15, Kb2, Kb4, Kh3-4, Kh6, Kh8, Kt1, Kt4-5, Kt7, On4, On6-7, Pi4, Pi6, P14-5, P17-8, Pr1, Pr3, Pr9-10, Pr12, Pr16-17, Sh2, Sh4, Ve1, Ve3, Ve5, Vi2, Vi5, Vi7-8, Vt3-4, Vt6.

Catarhoe rubidata (Den. \& Schiff.). Kt1 (Krulikovsky 1909).

Camptogramma bilineata (L.). Kb2, Kt1, Vt3.

Ochyria quadrifasciata (Cl.). Ka5, Kh15, Kt1, Pi4, Vt3.

* Orthonama vittata (Borkh.). Sh4.

Xanthorhoe abrasaria (H.-S.). So0.

X. annotinata (Zett.). Pi4, Pr2, Pr16, So6-7, Vi3.

$X$. decoloraria (Esp.). Pi4, P12.

*X. designata (Hufn.). Pr1, Pr16.

X. ferrugata (Cl.). Kh12, Kt1, Pi4, P13, Pr1, Pr9, Pr16, So1.

X. fluctuata (L.). Kh2, Kt1, Me3, Pi2, Pr1, Pr12, So1. 
X. montanata (Den. \& Schiff.). Ka1, Ka8-9, Ka11-14, Kb4, Kh2, Kh9, Kh12, Kh15, Ko2-3, Kt1-2, Pi4, P12-3, Pr1, Pr9, Pr16-17, So1, So4-6, Us3-4, Us6, Ve1, Ve4, Vi6.

X. spadicearia (Den. \& Schiff.). Kh12, Ko3, Me3, Pi2, Pi4, P12, Pr2, Pr16, Sh2, So4-6, Ve8.

*Euphyia unangulata (Haw.). Ka13, Ko3, Us6.

Epirrhoe alternata (Müller). Kh16, Pi4, P13, So6, Us3.

*E. pupillata (Thnbg.). Pr28.

E. tristata (L.). Ka8, Ka14, Kh2, Ko3, Kt2, Pi4, P13, Pr9, Pr16, Us2, Us4, Ve4, Ve6.

*Earophila badiata (Den. \& Schiff.). Kh15.

* Anticlea derivata (Den. \& Schiff.). Kh15, P13.

Mesoleuca albicillata (L.). Ka11, Ka13, Kh16, Pi4, P12, Pr1, Pr16, Us3, Us5.

Pelurga comitata (L.). Ka1, Kh15, Kt1, Pr1, Vi3.

Entephria caesiata (Den. \& Schiff.). Kt1, Pi4, P12, Pr1, Pr10, Pr16, Pr24, So1, So7.

Spargania luctuata (Den. \& Schiff.). Pi4, P13, Pr16, So6.

Hydriomena furcata (Thnbg.). Ka1, Ka15, Pi4, P12-3, Pr1, Pr6, Pr9, Pr12, Pr16, Sh4, Vi3.

H. impluviata (Den. \& Schiff.). Pi4, P12-3, Pr12, Pr16, Pr24, So1.

H. ruberata (Freyer). P12-3, Pr2, Pr16, Ve9.

Colostygia aptata (Hbn.). Pi4 (Antonova \&

Tikhomirov 2002).

C. pectinataria (Knoch). Ko2-3, Kt1, P13, Pr16. Electrophaes corylata (Thnbg.). Kt1, Pi4, So6-7.

*Chloroclysta miata (L.). So1.

Dysstroma citrata (L.). Ka15, Kt1, Pi4, P12, Pr1, Pr12, Pr16, Pr28, Sh2, Vi3.

*D. latefasciata (Stgr.). Pi4.

D. truncata (Hufn.). Kh6, On4, Pi4, Pi6, P12, P14, Pr9-10, Pr15-16, Sh4.

Cidaria fulvata (Forster). Pi4 (Antonova \& Tikhomirov 2002).

Plemyria rubiginata (Den. \& Schiff.). Pi4, Sh4.

Thera juniperata (L.). P12, Pr16.

Heterothera serraria (Lienig \& Z.). P12, Ve7.

Eulithis mellinata (F.). Kt1 (Krulikovsky 1909).

E. populata(L.). Kh8, Kh15, Kt1, Me2, On3, Pi4,

P12, P15, Pr1, Pr9, Pr16, Pr23, So1, Vi3.

E. prunata (L.). Kh15, Pi4, Pi6, P13, Pr1, Pr9,

Pr16-17, Pr23, Pr28, Sh4, Ve3, Vi3.

*E. pyropata (Hbn.). Ka1, Ka15.

E. testata (L.). Pi4, P12, Pr16, Vi3.
*Gandaritis pyraliata (Den. \& Schiff.). Kt3, Pr9, Vt4.

Ecliptopera capitata (H.-S.). Pi4 (Antonova \& Tikhomirov 2002).

E. silaceata (Den. \& Schiff.). Kh15, Pi2, Pi4.

Cosmorhoe ocellata (L.). Kt1 (Krulikovsky 1909).

*Lampropteryx otregiata (Metcalfe). Pr16.

*L. suffumata (Den. \& Schiff.). Pr1, Us2.

* Operophtera brumata (L.). Pr1.

* O. fagata (Scharfenberg). P12.

Epirrita autumnata (Borkh.). Kh15, Pr1, So1, Vi3.

*Asthena albulata (Hufn.). Vt6.

*Euchoeca nebulata (Scop.). Ka13, Ko3, P13, Pr16, Pr24, Ve1, Vi6.

*Venusia cambrica Curt. So6.

Hydrelia flammeolaria (Hufn.). Ka8, Ka13, Ko3, Kt1, Us3, Us6.

*H. sylvata (Den. \& Schiff.). Ka11.

Rheumaptera hastata (L.). Ka7, Ka13, Kh5, Kh12, Kt1, Me1, Pi2, Pi4, P12-6, P110, Pr1-2, Pr16, Pr28, So4, So6-7, Us2, Us6, Ve6.

Rh. subhastata (Nolcken). Me3, Pi4, Pr1-2, Pr16, So1, So6.

Hydria undulata (L.). Kh9, Kt1, Pi4, Pr16, Ve2. Coenocalpe lapidata (Hbn.). Kt1, Me2, Pi4, Vi3.

Horisme aemulata (Hbn.). Pi4 (Antonova \& Tikhomirov 2002).

Melanthia mandshuricata (Bremer). Pi4 (Antonova \& Tikhomirov 2002). This is the westernmost record of this Euro-Siberian species which was only recently reported as new to Europe from the South Ural Mts. (Ahola et al. 1997).

*M. procellata (Den. \& Schiff.). Kh6. This is the northernmost record of the species.

*Anticollix sparsata (Tr.). Ka8, Ka13.

Mesotype didymata (L.). Kt1, Pi4, P12, Pr1, Pr16, Sh4.

M. parallelolineata (Retz.). Pi4 (Antonova \& Tikhomirov 2002).

Perizoma albulata (Den. \& Schiff.). Ka10, Kh15, On5, Pi4, P13, Pr16, So4, So6-7, Ve4, Ve8.

P. alchemillata (L.). Kh15, Kt1, On1, P13, Pr1.

P. blandiata (Den. \& Schiff.). Pi4, Pr16, Vt3.

Martania taeniata (Steph.). Ka15, Pi4, P12-3, Pr16, Ve3.

Gagitodes sagittata (F.). Pi4 (Antonova \& Tikhomirov 2002). 
* Pasiphila chloerata (Mabille). Pr1.

*P. debiliata (Hbn.). Ka1, Pi6, Pr1, Pr16, Vi7.

P. rectangulata (L.). Kt1, Pr1, Pr16, Vi8.

Eupithecia abietaria (Goeze). Pi6, P12.

E. absinthiata (Cl.). Kt1 (Krulikovsky 1909).

E. actaeata Walderdorff. Pi4 (Antonova \& Tikhomirov 2002).

*E. analoga Djak. Pr16.

E. assimilata Doubleday. Pi4 (Antonova \& Tikhomirov 2002).

*E. conterminata (Lienig \& Z.). On1, Pr2, So6.

*E. exiguata (Hbn.). Ve1.

E. gelidata Möschl. Pi4 (Antonova \& Tikhomirov 2002).

*E. indigata (Hbn.). Pr2, Pr16.

E. intricata (Zett.). Ka14, Kh12, Pi4, Pr9, Pr16, Ve1.

E. lariciata (Freyer). Pi4 (Antonova \& Tikhomirov 2002).

*E. plumbeolata (Haw.). Kb4, Me3, P12, Pr1, Pr16, So7, Us3-4, Ve3-4.

E. pusillata (Den. \& Schiff.). Ka15, Pi4, Pr1, Pr16, Vi3.

*E. pygmaeata (Hbn.). P12, Pr16, So4, So6, Us4, Us6.

E. satyrata (Hbn.). Ka10, Pi4, P12-3, Pr1-2, Pr9, Pr16, So4-6, Us2, Ve6.

E. sinuosaria (Ev.). Kt1 (Krulikovsky 1909).

E. subfuscata (Haw.). Kt1, Ny1, Pi4, Pr9, Pr16.

*E. subumbrata (Den. \& Schiff.). Ka14, Ve1.

E. succenturiata (L.). Ka15, Pi4, Pr12, Pr16, So6, Ve1, Vi3.

*E. tantillaria Boisd. So6.

*E. tenuiata (Hbn.). Pr16.

*E. valerianata (Hbn.). Pr16, Vi6.

E. virgaureata Doubleday. Ka14, Pi2, Pi4, Pr16, Ve1.

E. vulgata (Haw.). Kh9, Me3, Pi4, P12-3, Pr1, Pr16, So5-7.

Odezia atrata (L.). Kb2, Kb4, Kh7, Kt1, Pi4, P16, Pr15-16, So0, Ve5.

Carsia sororiata (Hbn.). Kh4, Kh6, Kh10, On3, Pi4, Pr1, Pr16, So2, So9, Ve2, Vt5.

* Aplocera praeformata (Hbn.). Sh4.

Lobophora halterata (Hufn.). Pr16 (Zelenova 1972).

*Pterapherapteryx sexalata (Retz.). Ka11-12, Pr1, Pr28.

Trichopteryx carpinata (Borkh.). Kh15, P13, Pr1, Pr16, So1.
Notodontidae

Clostera anachoreta (Den. \& Schiff.). Pr1, Pr16, Pr23.

C. curtula (L.). Pr1, Pr16.

C. pigra (Hufn.). Ka1, Pr1, Pr15-16.

Notodonta dromedarius (L.). Pr1, Pr16, Vi3.

N. torva (Hbn.). Pi4, Pl2, Pr1, Pr16.

N. tritophus (Den. \& Schiff.). Pr0 (Zelenova 1976).

N. ziczac (L.). Kt1, Pr1.

Pheosia gnoma (F.). Kt1, Pr1, Pr16.

Ph. tremula (Cl.). Ka15, Pr1.

Pterostoma palpina (Cl.). P13, Pr1, Pr16.

Ptilodon capucina (L.). Pi4, P13, Pr16, Vi3.

Odontosia carmelita (Esp.). Kt1 (Krulikovsky 1909), Pr16 (Zelenova 1972).

Furcula bifida (Brahm). P12, Pr16 (Zelenova 1972).

* Furcula furcula (Cl.). Pr1.

Cerura vinula (L.). Ka12, Kt1, P13, Pr1, Pr5, Pr16-18, Pr21.

Phalera bucephala (L.). P12, Pr1, Pr16.

Erebidae

Scoliopteryx libatrix (L.). Kh15, Kt1, Pi4, P12-3, Pr1, Pr16, Sh4, Ve9.

*Rivula sericealis (Scop.). Ka15, Kh8, Sh4, Vt6.

*Hypena crassalis (F.). Kh9, So6-7.

H. proboscidalis (L.). Ka15, Kb4, Kh15, On3, Pi4, Sh4.

H. rostralis (L.). Kt1 (Krulikovsky 1909).

Leucoma salicis (L.). Kh14, Pr1, Pr16, So1.

* Calliteara pudibunda (L.). Ka1, Ve1.

Orgyia antiqua (L.). Kt3, Pi4, P12, Pr1, Pr16, Vg1.

Dicallomera fascelina (L.). Kt1 (Krulikovsky 1909).

Spilosoma lubricipeda (L.). Ka1, Ka7, Kh16, Kt1, Pi4, Sh4, Ve1, Vi3.

S. lutea (Hufn.). Ka1, Ka15, Kt1.

Diacrisia sannio (L.). Ka12, Ko2, Kt1, Pi4, Pr8, Pr12, So4, So6-8, Ve8.

Pararctia lapponica (Thnbg.). Me1 (Poppius 1906).

Borearctia menetriesii (Ev.). Pi3 (Bolotov et al. 2013c).

Phragmatobia fuliginosa (L.). Kt1 (Krulikovsky 1909).

*Parasemia plantaginis (L.). Kh15, Pi4, P13, Pr16, So1. 
Tyria jacobaeae (L.). Kt1 (Tatarinov et al. 2003). This is the northernmost record of the species. Arctia caja (L.). Kh15-16, Kt1, Pi4, Pr1, Pr17, Pr28.

A. villica (L.). Ktl (Tatarinov et al. 2003). This is the northernmost record of the species.

* Miltochrista miniata (Forster.). Ka15, Sh4.

Cybosia mesomella (L.). Kt1, So7.

*Atolmis rubricollis (L.). Sh4.

*Eilema depressa (Esp.). Ka15, Kb4.

*E. griseola (Hbn.). Ka15.

*E. lutarella (L.). Kb4, Sh4, Vi3.

*E. sororcula (Hufn.). Us5.

Setina irrorella (L.). Kt1 (Krulikovsky 1909).

* Calyptra thalictri (Borkh.). Kh15, Vi3.

*Herminia tarsipennalis (Tr.). Ka11, Ka13, Ka15.

*H. tarsicrinalis (Knoch). Ka13, Us4.

Polypogon tentacularia (L.). Ka14, Kb4, Ko2, Kt1, Pi4, P12-3, Pr1, Pr16, Pr23, Us4, Ve1, Ve5, Ve8, Vi7, Vt3, Vt6.

*Pechipogo strigilata (L.). Ve1.

*Hypenodes humidalis Doubleday. Pr16, Ve2.

* Lygephila pastinum (Tr.). Pr12, Vi3.

Parascotia fuliginaria (L.). Kt1 (Krulikovsky 1909).

* Phytometra viridaria (Cl.). Pl3.

*Colobochyla salicalis (Den. \& Schiff.). Ka1, Kh12, Ko3, Us4, Ve4.

*Laspeyria flexula (Den. \& Schiff.). Kb2.

* Trisateles emortualis (Den. \& Schiff.). Us3.

Catocala adultera Ménétriés. Ka2, Kh7, Pi4, Pr1, Pr12.

C. fraxini (L.). Kh15, On0, Pi0, P19.

*C. fulminea (Scop.). Kh15, Sh4.

C. nupta (L.). Pr1 (Bolotov et al. 1998).

Euclidia mi (Cl.). Kh16, Kt1.

E. glyphica (L.). Ka7, Ka11-14, Kh8, Kh15, Ko2-3, Kt1, Pi4, Pr12, Pr16-17, Sh4, So1, So3-4, So6, Us3-6, Ve1, Ve4-5, Ve8.

Nolidae

Nola aerugula (Hbn.). Kt1 (Krulikovsky 1909).

*Nycteola degenerana (Hbn.). Ka1, Ka15, Kh3, Kh15, Vi3, Vt6.

N. revayana (Scop.). Kt1 (Krulikovsky 1909).

Noctuidae

*Abrostola tripartita (Hufn.). Vi3.

*Macdunnoughia confusa (Steph.). Ka1.
Diachrysia chrysitis (L.). Kh15, Kt1, Pi4, Vt3.

*D. stenochrysis (Warr.). Vi3, Vt3.

Polychrysia moneta (F.). Pi4, Pr1, Vi3.

*Lamprotes c-aureum (Knoch). Sh4.

* Autographa bractea (Den. \& Schiff.). Kt8.

*A. excelsa (Kretschmar). Ka1, Ka15, Vi3.

A. gamma (L.). Ka12, Kh15, Ko2, Kt1, Pi4, So1, So6-7, Ve1, Ve4.

*A. macrogamma (Ev.). Ar0.

*A. pulchrina (Haw.). Kh15, So1.

Syngrapha ain (Hochenw.). Pi4. This record hints that the westernmost populations of this species may occur in Arkhangelsk oblast, far more westwards than earlier records made near the Ural Mts. (Sinev 2008). In Fennoscandia, a single migrant specimen was recorded in Finland (St: Nakkila; Repo 1995).

S. interrogationis (L.). Ka2, Kh6, Kt1, Pi4, P12, So1, Ve1, Vi3, Vi7, Vt6.

Plusia festucae (L.). Ka1, Pi4.

Plusia putnami (Grote). Pr1.

*Deltote pygarga (Hufn.). Ka1, Ka5, Ka11, Ka13-15, Kb4, Ko2-3, Sh4, Us3, Ve4, Vt3.

*D. bankiana (F.). Ka1, Ka14, Ve4.

D. uncula (Cl.). Kt1, P13, Pr16.

*Acontia trabealis (Scop.). Vi3. This is most likely a migrant specimen, because it was collected in 2011, when hundreds of specimens originating presumably from SE part of European Russia were recorded in Finland (Hyönteistietokanta 2014).

Colocasia coryli (L.). P12, Pr16, Pr23, Vi6.

*Moma alpium (Osbeck). Sh4, Ve9.

*Acronicta alni (L.). Pr1, P17, Sh4.

A. auricoma (Den. \& Schiff.). Pr16, So1.

A. leporina (L.). P12, Pr1, Pr23.

*A. menyanthidis (Esp.). Ka1, Pr16.

*A. psi (L.). Sh4, Ve9.

A. rumicis (L.). Kt1, Sh4, Ve1, Ve9.

*A. strigosa (Den. \& Schiff.). Sh4, Ve9.

Subacronicta megacephala (Den. \& Schiff.). Pr1, Pr16, Pr23, Sh4, So0.

* Tyta luctuosa (Den. \& Schiff.). Ka1. This is most likely a migrant specimen, because it was collected in 2011, when hundreds of specimens originating presumably from SE part of European Russia were recorded in Finland (Hyönteistietokanta 2014).

Cucullia umbratica (L.). Kt1 (Krulikovsky 1909). 
Calophasia lunula (Hufn.). Kt1 (Krulikovsky 1909).

*Amphipyra perflua (F.). Ka15, Sh4, Vi3.

*A. tragopoginis (Cl.). Kh15-16, Pr12.

Pyrrhia exprimens (Walk.). Pi4 (A. Tikhomirov, pers. comm.).

Heliothis viriplaca (Hufn.). Kt1 (Krulikovsky 1909).

Caradrina clavipalpis (Scop.). Ka1, Kt1, Le4, Me1, Pi10, P13, Pr1-2, Pr16, So0, Ve1.

C. morpheus (Hufn.). Ka1, Kh16, Kt1, Ve1, Vi3.

C. petraea Tengstr. Kt1 (Krulikovsky 1909). This Euro-Siberian species occurs in eastern Estonia and Eastern Karelia (Jürivete \& Õnap 2008, Sinev 2008), but it has never been recorded in the Nordic countries.

* Athetis pallustris (Hbn.). P13.

*Enargia paleacea (Esp.). Ka2, Kh15, Pi4, P14, Pr12, Vi3.

*Ipimorpha retusa (L.). Vi3.

*Cosmia pyralina (Den. \& Schiff.). Ka1, Sh4, Vi3.

C. trapezina (L.). Kt1, Pr1, Vi3.

* Dypterygia scabriuscula (L.). Sh4.

* Hyppa rectilinea (Esp.). Pr16, So6, Ve9.

*Euplexia lucipara (L.). Ka1, Sh4.

Crypsedra gemmea (Tr.). Ka2, Pi4, Vi3.

*Staurophora celsia (L.). Vi3.

Celaena haworthii (Curt.). Le2, Pi4, Vi3.

*Helotropha leucostigma (Hbn.). Vi3.

Gortyna flavago (Den. \& Schiff.). Kt1, Vi3.

Hydraecia micacea (Esp.). Kh15-16, Kt1, Pi4, P12, Pr12, Sh4, Vi3.

*H. ultima Holst. Vi3.

Amphipoea fucosa (Freyer). Ka1-2, Pi4, Pr12, Pr16, Sh4, Vi3.

*A. lucens (Freyer). Vi3.

A. oculea (L.). Kt1, Pi4, Vi3.

* Rhizedra lutosa (Hbn.). Vi3.

* Phragmatiphila nexa (Hbn.). Pr12, Vi3.

*Denticucullus pygmina (Haw.). Vi3.

Apamea crenata (Hufn.). Kh15-16, Pr1.

*A. remissa (Hbn.). Pr1, Pr16, Sh4.

*A. scolopacina (Esp.). Ka15.

A. sordens (Hufn.). Kt1 (Krulikovsky 1909).

*A. unanimis (Hbn.). Ka1, Sh4, Ve9.

A. lateritia (Hufn.). Kt1, Pi4, Pr1, Sh4, Vi3.

A. monoglypha (Hufn.). Ka1, Kt1, Sh4.

*Lateroligia ophiogramma (Esp.). Ka1, Vi3.

*Mesapamea secalis (L.). Ka2, Sh4.
*Mesoligia furuncula (Den. \& Schiff.). Ka1, Sh4.

*Oligia strigilis (L.). Sh4.

Brachylomia viminalis (F.). Ka2, Ka15, Pi4, Pl2, Pr1, Pr16.

Parastichtis suspecta (Hbn.). Ka15, Kt1, Kt4, Pi4, P12, Pr1, Pr16, Vi3.

Xanthia togata (Esp.). Kh16, Pi4, Pr16, Vi3.

$X$. icteritia (Hufn.). Kh15, Pi4, P12, Pr16, Vi3.

Agrochola helvola (L.). On0, Pi4, Pr16, Vi3.

Hillia iris (Zett.). Kt1, Pi4, So1

*Lithophane consocia (Borkh.). Pr1, Vi3.

L. lamda (F.). P12.

L. socia (Hufn.). Kt1, Pr1.

Xylena solidaginis (Hbn.). Ka2, Kh15, Pi4, P12$3, \operatorname{Pr} 1$.

$X$ vetusta (Hbn.). Kh15, P12, Pr1.

Eupsilia transversa (Hufn.). Kt1 (Krulikovsky 1909).

Antitype chi (L.). Ka2, Kt1, Pi4.

Blepharita amica (Tr.). Kh16, Kt1, Vi3.

Orthosia gothica (L.). Pr1, Pr16, So1.

* Tholera cespitis (Den. \& Schiff.). Vi3.

Th. decimalis (Poda). Kt1 (Krulikovsky 1909).

Cerapteryx graminis (L.). Ka3, Pi4, P12, Pr1, Pr11, Pr16, Pr24, Sh4, Vi3.

*Anarta myrtilli (L.). So4.

A. trifolii (Hufn.). Ka1, Kt1, Vi3.

*Coranarta cordigera (Thnbg.). Pi10, Pr1.

Polia bombycina (Hufn.). Kt1, Sh4, Ve9.

*P. nebulosa (Hufn.). Sh4, Ve9.

* Lacanobia contigua (Den. \& Schiff.). Ka1.

*L. oleracea (L.). Ka1, Sh4.

L. suasa (Den. \& Schiff.). Kt1, Pr1.

${ }^{*}$ L. thalassina (Hufn.). Pr1, Pr16, Sh4, Us6, Ve1, Ve9.

*Melanchra persicariae (L.). Sh4, Ve9.

Ceramica pisi (L.). Kh9, Pi4, P12, Pr1, Pr16, Ve1.

Papestra biren (Goeze). Pr16.

Hada plebeja (L.). Ka1, Kt1, P12-3, Pr1, So0, Ve1.

Mamestra brassicae (L.). Kt1 (Krulikovsky 1909).

*Sideridis reticulata (Goeze). Ve1.

S. rivularis (F.). Kt1 (Krulikovsky 1909).

Hecatera bicolorata (Hufn.). Kt1 (Krulikovsky 1909).

Mythimna conigera (Den. \& Schiff.). Ka1, Kt1.

M. impura (Hbn.). Ka1, Kh3, Kh16, Pi4, Sh4, Vi3, Vt3, Vt4.

Leucania comma (L.). Kh16, Kt1, Pr25. 
Lasionycta imbecilla (F.). Kt1, Sh4.

Euxoa nigricans (L.). Kt1 (Krulikovsky 1909).

E. recussa (Hbn.). Kt1 (Krulikovsky 1909), Pi4

(A. Tikhomirov, pers. comm.).

E. tritici (L.). Kt1 (Krulikovsky 1909).

Agrotis clavis (Hufn.). Ktl (Krulikovsky 1909).

A. exclamationis (L.). Ka1, Kt1, Sh4, Ve1.

A. segetum (Den. \& Schiff.). Kt1 (Krulikovsky 1909).

* Axylia putris (L.). Sh4, Ve1.

Ochropleura plecta (L.). Kt1, Sh4.

* Diarsia dahlii (Hbn.). Ve9, Vi3.

D. mendica (F.). Kt1, Sh4, So6.

*D. rubi (Vieweg). Kh16.

Rhyacia simulans (Hufn.). Kt1 (Krulikovsky 1909).

Chersotis cuprea (Den. \& Schiff.). Ka3, Pi4, P17, Pr16, Sh4, So1, Vg1, Vi3.

* Cryptocala chardinyi (Boisd.). P17.

Spaelotis ravida (Den. \& Schiff.). Kt1 (Krulikovsky 1909).

Eurois occulta (L.). Ka1, Kh15, Kt1, On0, P13, Pr1, Pr16, Sh4, Ve9, Vi3.

Graphiphora augur (F.). Kh13, Kh15-16, P13, Pr1, Pr12, Sh4.

Anaplectoides prasina (Den. \& Schiff.). Pi4, Sh4.

Xestia alpicola (Zett.). Pi4 (A. Tikhomirov, pers. comm.).

X. baja (Den. \& Schiff.). Ka1-2, Kt1, Pi4, P14, Sh4, Vi3.

*X. ditrapezium (Den. \& Schiff.). Ka1, Sh4, Vi3.

This is probably an expansive species. In Finland, the species has demonstrated a remarkable expansion with over 70 exx. reported since its first discovery in 2006 and having now reached as far north as $K b$ : Ilomantsi (Hyönteistietokanta 2014).

X. rhaetica (Stgr.). ssp. fennica (Brandt). Pi4, Pr10.

*X. sexstrigata (Haw.). Vi3, Sh4.

X. speciosa (Hbn.). Pi4, P12.

*X. triangulum (Hufn.). Vi3.

* Protolampra sobrina (Dup.). Sh4.

\section{Excluded species}

Dahlica lichenella (L.). Kt1 (Krulikovsky 1909). This record is very uncertain and, most likely, is based on misidentification of D. lazuri (Cl.) which is the most common species. In Finland, $D$. lichenella is the rarest species of the genus and is found mainly on sea shores.

Ypsolopha ustella (Cl.), mentioned by Zelenova (1976) as Cerostoma radiatella Don., was reared from birch in Pr1. Since $Y$. ustella is a more southern species whose larvae feed on oak, we presume that this report actually refers to Y. parenthesella (L.), which is common in the area.

Acleris ferrugana (Den. \& Schiff.), mentioned by Zelenova (1976) as A. tripunctana Hb., had been reared from birch in Pr1. We attribute this record to $A$. notana (Don.), which is often confused with $A$. ferrugana.

Catoptria myella (Hbn.). Kt1 (Krulikovsky 1909). This record is very uncertain and, most likely, is based on misidentification of $C$. permutatella (H.-S.). Catoptria myella is generally confined to mountain regions of Central Europe, and Slamka (2008) questioned correctness of identifications on which the records from the northern Russia (Sinev 2008) are based.

Apatura iris (L.). Ar0 (Andreev 1995). Neither this species, nor the two following species have been recorded in Arkhangelsk oblast so far. Most likely, they were included into the cited publication on the basis of extrapolation of the species' distribution in Europe.

Coenonympha hero (L.). Ar0 (Andreev 1995). See the comment to Apatura iris.

Euplagia quadripunctaria (Poda). Ar0 (Andreev 1995). See the comment to Apatura iris.

Catocala elocata (Esp.). P12 (Zelenova 1972). This record is presumably based on a misidentified specimen, which had not been found in collections by L. F. Zelenova. The northernmost record in Russia is from St. Petersburg region (A. Matov, pers. comm.); in Finland the speceis is reported from $N$ only (Kullberg et al. 2014).

\section{Discussion}

This first regional checklist of moths and butterflies of Arkhangelsk oblast includes 1,036 species (538 species of microlepidoptera and 498 species of macrolepidoptera), 496 of which are 
recorded from this area for the first time. Eight species reported from the oblast in earlier publications are excluded from the list. The records of 929 species are based on the material from 160 localities that was examined by the authors. We have not seen any specimens of 107 species reported from Arkhangelsk oblast in earlier publications. In line with the earlier practice (Kozlov \& Jalava 1994) we consider these records as requiring confirmation. For seven species we do not have any information on sampling locality even at the level of an administrative unit within Arkhangelsk oblast. Six of these species were found in non-labelled student's samples in NArFU and one species was referred to in a publication that did not indicate exact sampling sites.

Although the fauna of Arkhangelsk oblast is clearly poorer than that of the Ural region due to the lack of higher mountain chains, it includes some Siberian taiga species which do not reach Fennoscandia. Also the northern distribution limits of several species extend further north in Arkhangelsk oblast than in the more western parts of Europe. A more continental climate is the likely reason for this pattern in some butterflies and larger moths. Similar patterns are seen in the distributions of several species of butterflies in Sweden and Finland, i.e. in the eastern regions they reach more northern latitudes. However, for other species the limited distribution can be explained by the current distribution of host plants as in the case of Syngrapha ain and Cydia zebeana feeding on larch, continuous distribution of which lies well on the eastern side of Onega Lake. Similarly, the distribution of Melanthia spp., Thyris fenestrella and Horisme aemulata depends on Clematis sibirica, which is abundant in the taiga east of Onega Lake.

From our point of view, the most interesting species in our list are those which do not occur in either the Baltic countries or Fennoscandia (including the Russian Karelia). Twenty-three species recorded in Arkhangelsk oblast are not found in Finland. However, the following comparison is restricted to well known groups in order to get a reliable picture of the differences between the regions.

Six species of butterflies and skippers found in Arkhangelsk oblast are not found in Finland, and two more species (Cupido alcetas, Erebia euryale) have been recorded in Finland only once. The families Erebidae, Lasiocampidae, Sphingidae and Noctuidae each had one species not found in Finland: Arctia villica, Phyllodesma japonica, Smerinthus caecus and Caradrina petraea, respectively; Geometridae add two species, Melanthia procellata and M. mandshuricata, to this list. Several of these species, in particular A. villica, P. japonica and C. petraea, have not demonstrated range expansion in northern Europe: they have been known to occur only few tens of kilometres SE of the Finnish-Russian border (Kaisila 1962, Marttila et al. 1996) for decades, but never expanded to Finland, although there is no limiting food plant or biotope preference as in the Pulsatilla and Clematis feeding species of Melanthia. Among butterflies, Neptis rivularis could have potential to disperse in rural areas in Finland where its food plants (Spirea spp.) are commonly cultivated. Also Leptidea morsei, which we found amazingly common in the Arkhangelsk oblast, may further expand westwards.

The composition of butterfly fauna hints that we may expect clearly more "exotic" southern and eastern species to be found in Arkhangelsk oblast, especially among microlepidoptera, as some interesting species have already popped up. The most striking faunistic record is Gnorimoschema robustella, previously known from the lowlands of the South Ural region only, but also findings of Caryocolum leucomelanella and Dichrorampha sequana are surprising as they lie quite far north of the known distribution ranges.

Clouded Apollo (Parnassius mnemosyne) is one of a few species of butterflies, the ecology and distribution of which is well studied in Arkhangelsk oblast (Rykov 2009, Bolotov et al. 2013b). We discovered several populations of $P$. mnemosyne in the southern part of the region (localities Ko2 and Us3-5), where this species had not previously been recorded. In all these localities, the species was common on meadows along the riverbanks: one observer recorded 1 to 12 specimens in each habitat (400 to $2,500 \mathrm{~m}^{2}$ ) during 45-60 min.

We classify the current level of knowledge of the lepidopteran fauna of Arkhangelsk oblast as modest. This conclusion is based on the comparison with the well-known fauna of Finland, which 
amounts about 2,600 species (J.K., pers. obs.), and on the ratio between the numbers of collected micro- and macrolepidoptera (1.08) which is much lower than the value of 1.6 that is characteristic of well-studied faunas of boreal forest zone (Sinev 2008). However, the Arkhangelsk oblast lies outside the distribution range of numerous woody plants that are naturally growing in the hemiboreal zone in Finland, which excludes dozens of moth species from its fauna. Particularly, oak (Quercus robur), maple (Acer platanoides), lime (Tilia spp.), common buckthorn (Rhamnus catharctica) and blackthorn (Prunus spinosa) do not belong to the native flora of Arkhangelsk oblast, although some of these species are cultivated in urban parks. The lesser extent of the study region towards the North relative to Finland and absence of mountains further remove several arctic and montane species from the potential fauna. Still we estimate that 500 to 800 species of Lepidoptera remain to be found in the Arkhangelsk oblast.

Among the districts of Arkhangelsk oblast (Fig. 1), the fauna of the surroundings of Arkhangelsk is documented much better than the fauna of other regions, with 385 species recorded from Malye Karely (Pr16; 28 km S of Arkhangelsk), the favourite collecting site of L. F. Zelenova. In contrast, the fauna of south-western and northeastern parts of the oblast remains clearly underexplored. In terms of phenological groups, spring, early summer and late autumn species are less recorded than mid-summer species.

Acknowledgements. We are thankful to B. Filippov, S. Sinev and V. Tsvetkov for numerous practical arrangements and to I. Bochneva for operating light trap in Vi3. We are indebted to I. Bolotov, A. Tikhomirov, A. Popov, M. Tähtinen, J. Paukkunen and E. Tsvetkov for permission to use their materials and/or unpublished data, to L. Kaila, S. Sinev, A. Lvovsky, V. Mironov, A. Matov and E. J. van Nieukerken for identification of difficult taxa, to I. Bolotov, B. Filippov, M. Podbolotskaya and L. Zelenova for providing additional information and to L. Kaila, J. Junnilainen and M. Klepikov for helpful comments to an earlier draft of the manuscript. This work was supported by the Finnish Lepidopterological Society, Academy of Finland (project 122133 and researcher exchange grants), Percy Sladen Memorial Fund, and by a strategic research grant from the University of Turku.

\section{References}

Ahola, M., Kaitila, J.-P., Nupponen, K., Junnilainen, J., Olshwang, V. \& Mikhailov, Yu. E. 1997: Macrolepidoptera. - In: Olswang, V., Bogacheva, I., Nikolaeva, N., Mikhailov, Yu., Gorbunov, P. \& Zinovjev, E. (eds.): Achievments of Entomology in the Urals: 98104. Ural State University, Ekaterinburg. 208 pp. [In Russian.]

Andreev, V. A. (ed.) 1995: Red Book of Arkhangelsk oblast. - Committee for Protection of Environment and Natural Resources, Arkhangelsk. 329 pp. [In Russian.]

Antonova, E. M. \& Tikhomirov, A. M. 2002: Geometrid moths (Lepidoptera, Geometridae) of the Pinezhsky Nature Reserve. - Bulletin of Moscow Society of Naturalists, Biological Series 107(6): 38-41. [In Russian, English abstract.]

Bolotov, I. N. 2002: Butterflies (Lepidoptera, Rhopalocera) of Arkhangelsk city and its vicinities. - Zoologichesky Zhurnal 81: 457-462. [In Russian, English abstract.]

Bolotov, I. N. \& Semushin, A. V. 2003: (Protected species of invertebrates of Pinega - Severnaya Dvina interfluve. Ecological and faunistic inventory.) - Ural Branch, Russian Academy of Sciences, Yekaterinburg. 83 pp. [In Russian.]

Bolotov, I. N., Gofarov, M. Yu. \& Shvakov, Yu. A. 1998: Rare moths (Lepidoptera, Metaheterocera) from surroundings of Arkhangelsk. - In: Taskaeva, L. G. (ed.), Topical problems of ecological education and environmental protection: Abstracts of presentations made at II international scientific and practical conference: 90-91. ELPA publ., Arkhangelsk. 131 pp. [In Russian.]

Bolotov, I. N., Podbolotskaya, M. V., Kolosova, Yu. S. \& Zubrii, N. A. 2013a: The current flow of migrants and its contribution to butterfly faunas (Lepidoptera, Rhopalocera) on marine islands with young allochthonous biota. - Biology Bulletin 40: 78-88.

Bolotov, I. N., Gofarov, M. Yu., Rykov, A. M., Frolov, A. A. \& Kogut, Y. E. 2013b: Northern boundary of the range of the Clouded Apollo butterfly Parnassius mnemosyne (L.) (Papilionidae): climate influence or degradation of larval host plants? — Nota Lepidopterologica 36: 19-33.

Bolotov, I. N., Gofarov, M. Yu., Kolosova, Yu. S. \& Frolov, A. A. 2013c: Occurrence of Borearctia menetriesii (Eversmann, 1846) (Erebidae: Arctiinae) in Northern European Russia: a new locality in a disjunct species range. - Nota Lepidopterologica 36: 65-75.

Burak, S. V. \& Ezhov, O. N. 2011: (Pests and diseases of planted trees and shrubs in Arkhangelsk region.) Plant Protection News 2011(1): 46-50. [In Russian.]

Byzova, N. M. (ed.) 2007. Pomor Encyclopaedia. Vol. 2. Nature of the Arkhangelsk North. - Pomor University, Arkhangelsk. 603 pp. [In Russian].

Danilevsky, A. S. \& Kuznetzov, V. I. 1968: (Leaflollers Tortricidae. Triba Laspeyresiini. Fauna of the 
U.S.S.R, Lepidoptera, vol. 5, pt. 1.) - Nauka, Leningrad. 635 pp. [In Russian].

Ezhov, O. N. 2008: (Pests and diseases of urban greenery in Arkhangelsk industrial hub.) - Lesnoi Zhurnal (Forest Journal), 2008(3): 45-50. [In Russian.]

Ezhov, O. N. \& Burak, S. V. 2010: (Pests and diseases of trees and shrubs in arboretum of Arkhandgelsk state technical university.) — Plant Protection News 2010(2): 50-54. [In Russian.]

Filippov, B. Y. 2009: Specific characteristics of insect fauna of nearly undisturbed forests of Pinega - Severnaya Dvina interfluve. - In: Kauhanen, H., Neshataev, V., Huhta, E. \& Vuopio, M. (eds.), Coniferous forests of Northern latitudes - from investigation to ecologically responsible forest management: 137-143. The Finnish Forest Research Institute, Jyväskylä. 172 pp. [In Russian.]

Gustafsson, B. 2014: Svenska fjärilar - Lepidoptera [www document]. URL http://www2.nrm.se/en/svenska_fjarilar/svenska_fjarilar.html. (Site visited on 14 January, 2014).

Haarto, A. \& Winqvist, K. 2006: Finnish flies of the family Therevidae. - Entomologica Fennica 17: 46-55.

Huemer, P. \& Karsholt, O. 2010: Gelechiidae II (Gelechiinae: Gnorimoschemini). (Microlepidoptera of Europe. Vol. 6). - Apollo Books, Stenstrup. 586 pp.

Hyönteistietokanta 2014: Lepidoptera [www document]. URL http://hyonteiset.luomus.fi/insects/main/EntDatabase.html\#search-LEP. (Site visited on 14 January, 2014).

Itämies, J., Mutanen, M. \& Mutanen, T. 1996: Lampronia standfussiella (Zeller, 1839) (Lepidoptera: Prodoxidae), Suomelle uusi pikkuperhoslaji. - Baptria 21: 119-121.

Jürivete, U. 2012: Eesti faunale uued pisiliblikad (Microlepidoptera) aastail 2010-2011. Lepinfo. Lk. 6.

Jürivete, U. \& Õunap, E. 2008: Eesti liblikad. Estonian Lepidoptera: Catalogue. - Tallinn, Eesti Lepidopteroloogide Selts, $175 \mathrm{pp}$.

Kaisila, J. 1962: Immigration und Expansion der Lepidopteren in Finnland in den Jahren 1869-1960. —Acta Entomologica Fennica 18: 1-452.

Karsholt, O., Nieukerken, E. J. van \& Jong, Y. S. D. M. de. 2013: Lepidoptera, Moths. - Fauna Europaea version 2.6.2 [www document]. URL http://www.faunaeur.org. (Site visited on 14 January, 2014).

Kolev, Z. \& Kullberg, J. 2000: Cupido alcetas (Hoffmansegg, 1804), a new bluewing species to Finland with comments on its distribution in Europe. - Baptria 25(4): 157-162. [In Finnish, English summary.]

Kozlov, M. V. \& Jalava, J. 1994: Lepidoptera of Kola Peninsula, Northwestern Russia. — Entomologica Fennica 5: 65-85.

Kozlov, M. V. \& Kullberg, J. 2006: Lepidoptera of Terskij sea shore of the Kola Peninsula, Northwestern Russia. — Entomologica Fennica 17: 123-129.

Krulikovsky, L. 1906: Beitrag zur Lepidopterenfauna des Gouvernements Wologda. - Societas Entomologica 20: $153-156$.

Krulikovsky, L. 1909: To the fauna of Lepidoptera of Gou- vernement Vologda. - Russkoe Entomologicheskoe Obozrenie 9: 65-79. [In Russian.]

Kullberg, J., Albrecht, A., Kaila, L. \& Varis, V. 2002: Checklist of Finnish Lepidoptera - Suomen perhosten luettelo. - Sahlbergia 6(2): 45-190.

Kullberg, J., Albrecht, A., Kaila, L. \& Varis, V. 2014: Checklist of Finnish Lepidoptera - Suomen perhosten luettelo. — [www document]. URL http://koivu.luomus.fi//elaintiede/hyonteiset/perhoset/ (Site visited on 18 January, 2014).

Lvovsky, A. L. \& Morgun, D. V. 2007: Butterflies of Eastern Europe. - KMK Scientific Press, Moscow. 443 pp. [In Russian.]

Marttila, O., Saarinen, K., Haahtela, T. \& Pajari, M. 1996: Suomen kiitäjät ja kehrääjät. — Kirjayhtymä, Helsinki. 384 pp.

Mazur, M. \& Kubisz, D. 2013: Distribution and migration of the xerothermic beetles (Coleoptera) in the Vistula River valley. - Monografie Faunistyczne 26: 4-245.

Nieukerken, E. J. van, Kaila, L., Kitching, I. J., Kristensen, N. P., Lees, D. C., Minet, J., Mitter, C., Mutanen, M., Regier, J. C., Simonsen, T. J., Wahlberg, N., Yen, S.H., Zahiri, R., Adamski, D., Baixeras, J., Bartsch, D., Bengtsson, B. E., Brown, J. W., Bucheli, S. R., Davis, D. R., De Prins, J., De Prins, W., Epstein, M. E., Gentili-Poole, P., Gielis, C., Hättenschwiler, P., Hausmann, A., Holloway, J. D., Kallies, A., Karsholt, O., Kawahara, A., Koster, S. (J. C.), Kozlov, M. V., Lafontaine, J. D., Lamas, G., Landry, J.-F., Lee, S., Nuss, M., Penz, C., Rota, J., Schmidt, B. C., Schintlmeister, A., Sohn, J. C., Solis, M. A., Tarmann, G. M., Warren, F. D., Weller, S., Yakovlev, R., Zolotukhin, V. \& Zwick, A. 2011: Order Lepidoptera Linnaeus, 1758. — In: Zhang, Z.-Q. (ed.), Animal biodiversity: An outline of higher-level classification and survey of taxonomic richness. - Zootaxa 3148: 212-221.

Poppius, B. R. 1906: Beiträge zur Kenntnis der Lepidopteren-Fauna der Halbinsel Kanin. - Acta Societatis pro Fauna et Flora Fennica 28(3): 1-11.

Repo, S. 1995: Makrotiedonannot 1994. — Baptria 20(1): 23-29.

Rykov, A. M. 2009: Recent distribution of the Clouded Apollo (Driopa mnemosyne) in Arkhangelsk oblast. - In: Taskaev, A. I. (ed.), Problems of investigation and conservation of the animal world in the North: Proceedings of the All-Russian scientific conference with international participation (Syktyvkar, Komi Republic, Russia, 16.-20. November 2009): 370-373. Komi Science Centre, Syktyvkar. 387 pp. [In Russian.]

Savenkov, N. \& Šulcs, I. 2010: Latvijas taurinii. Katalogs. — Eesti Lepidopteroloogide Selts, Tallinn. 176 pp.

Sinev, S. Yu. (ed.) 2008: Catalogue of the Lepidoptera of Russia. - KMK Scientific Press, St. Petersburg \& Moscow. 424 pp. [In Russian.]

Slamka, F. 2008: Pyraloidea of Europe, vol. 2: Crambinae and Schoenobiinae. - F. Slamka, Bratislava. 224 pp.

Svensson, I. 2005: Anmärkningsvärda fynd av småfjärilar (Microlepidoptera) i Sverige 2004. — Entomologisk Tidskrift 126: 21-33. 
Tatarinov, A. G. \& Dolgin, M. M. 1999: Diurnal Lepidopterans. (Fauna of the Northeastern Part of European Russia, vol. 7, pt. 1.) — Nauka, St. Petersburg. 183 pp. [In Russian.]

Tatarinov, A. G., Sedykh, K. F. \& Dolgin, M. M. 2003: Higher Heterocera. (Fauna of the Northeastern Part of European Russia, vol. 7, pt. 2.) - Nauka, St. Petersburg. 223 pp. [In Russian.]

Tengström, J. M. J. af. 1869: Catalogus Lepidopterorum faunae Fennicae praecursorius. - Notiser ur Sällskapets pro Fauna och Flora Fennica Förhandlingar 10: 287-371.

Tikhomirov, A. M. 1994: (Overview of Lepidoptera of Pinezhsky reserve.) - In: Egorov, V. N. (ed.), Ivanovo State University - regional centre of science, culture and education. Abstracts of presentations at jubilee scientific conference, 11.-12. January 1994: 251. Ivanovo State University, Ivanovo. 316 pp. [In Russian.]

Tikhomirov, A. M. \& Bolotov, I. N. 2000: (Fauna of butterflies (Lepidoptera, Rhopalocera) of Pinezhsky reserve and adjacent areas.) - In: Yudakhin, F. N. (ed.), North: Ecology: 334-342. Ural Branch, Russian Academy of Sciences, Yekaterinburg. 413 pp. [In Russian.]

Tuzov, V. K., Bogdanov, P. V., Devyatkin, A. L., Kaabak, L. V., Korolev, V. A., Murzin, V. S., Samodurov, G. D.
\& Tarasov, E. A. 1997: Guide to the butterflies of Russia and adjacent territories. Vol. 1. Hesperiidae, Papilionidae, Pieridae, Satyridae. — Pensoft Publ., Sofia \& Moscow. 480 pp.

Välimäki, P., Mutanen, M., Mutanen, T. \& Lehto, T. 2009: Mielenkiintoiset perhoshavainnot ja vaelluskatsaus 2008. - Baptria 34(2): 43.

Zelenova, L. F. 1972: Dendrophagous moths and butterflies (Lepidoptera) of Arkhangelsk and its surroundings. — Entomologicheskoe Obozrenie 51: 806-814 [In Russian, English abstract.]

Zelenova, L. F. 1973: (Dendrophagous leafrollers (Lepidoptera, Tortricidae) of Arkhangelsk and its surroundings.) - In: Koposova, T. S. (ed.), Issues of biology in education at universities and schools: 59-70. Vologda State Pedagogical Institute, Arkhangelsk. 127 pp. [In Russian.]

Zelenova, L. F. 1976: (Birch-feeding moths and butterflies (Lepidoptera) of Arkhangelsk and its surroundings.) - In: Bolotnikov, A. M. (ed.), Modern problems of zoology and improvement of the methods of its teaching in universities and schools. Abstracts of AllUnion scientific conference of zoologists from pedagogical universities.): 70-72. Perm State Pedagogical Institute, Perm. 378 pp. [In Russian.] 